\title{
Simple Broadband Measurements of Balanced Loads Using a Network Analyzer
}

\author{
M.W. van Rooyen
}

Thesis presented in partial fulfillment of the requirements for the degree of Master of Science in Electronic Engineering at the

University of Stellenbosch

Study Leader : K.D. Palmer

March 2001 


\section{- Declaration -}

"I, the undersigned, hereby declare that the work contained in this thesis is my own original work and that I have not previously in its entirety or in part submitted it at any university for a degree."

M.W. van Rooyen 


\section{Abstract}

Balanced loads such as antennas normally require baluns when they are measured. For wide band applications, designing and building a balun complicates the measurement and introduces errors. A simple model for load impedances was developed, together with a novel measurement procedure. The procedure enables the measurement of balanced loads using a network analyzer with no balun. Measured and simulated results are presented. 


\section{Opsomming}

Gebalanseerde laste soos antennas benodig gewoonlik balons om korrek gemeet te word. Die ontwerp van 'n balon vir wye band toepassings bemoeilik die metings en veroorsaak foute. 'n Eenvoudige model vir die lasimpedansies is ontwikkel sowel as 'n eenvoudige meetmetode. Die metode word gebruik om die gebalanseerde laste te meet met 'n netwerk analiseerder sonder die gebruik van 'n balon. Gemete en gesimuleerde resultate word getoon. 


\section{Contents}

$\begin{array}{ll}\text { Abstract } & 3\end{array}$

$\begin{array}{ll}\text { Opsomming } & 4\end{array}$

$\begin{array}{llr}1 & \text { Introduction } & 10\end{array}$

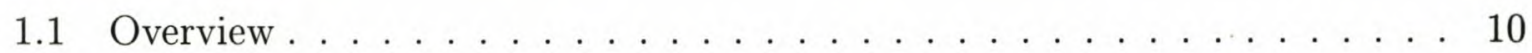

1.2 Current performance limitations of baluns . . . . . . . . . . . . 10

1.3 Research to date in eliminating the need for a balun . . . . . . . . . . . . 11

2 The proposed measurement system $\quad 12$

2.1 The proposed impedance model of an antenna . . . . . . . . . . . 12

2.2 The physical model . . . . . . . . . . . . . . . . . 12

2.3 Measurement procedure . . . . . . . . . . . . . . . . 13

2.4 Removing the effect of the semi rigid cables . . . . . . . . . . . . 13

3 Initial measured and simulated results $\quad 16$

3.1 The semi-rigid cables . . . . . . . . . . . . . . 16

3.2 Spiral antenna . . . . . . . . . . . . . . . 16

3.3 Dipole antenna . . . . . . . . . . . . . . . . . . 23

3.3.1 Confirmation of the common mode impedance model . . . . . . . 23

4 Sensitivity analysis $\quad 26$

4.1 The sensitivity equation . . . . . . . . . . . . . 26

4.2 Obtaining the sensitivity equation for the antenna impedances in terms of the measured S-parameters. . . . . . . . . . . . . . . . 27

4.3 Sensitivity analysis of the measured data . . . . . . . . . . . . 28

4.3.1 The sensitivity of the dipole antenna impedances in terms of the measured S-parameters . . . . . . . . . . . . . . . 29 
4.3.2 The sensitivity of the spiral antenna impedances in terms of the measured S-parameters . . . . . . . . . . . . . . . . . 29

4.3.3 The sensitivity of the impedances to the semi-rigid cables . . . . . 29

4.3.4 Height of the DUT above the EMC box . . . . . . . . . . . 36

4.3.5 Stray inductance at the connection point with DUT . . . . . . . . 37

4.4 Sensitivity analysis over a parameter range . . . . . . . . . . . . . 37

4.4.1 Choosing practical values for the analysis . . . . . . . . . . 37

4.4.2 The sensitivities for the S-parameters and the semi-rigid cables . . . 38

5 Interpretation of sensitivities and improvements 42

5.1 Interpretation of the sensitivity analysis of the measured data . . . . . . 42

5.1.1 The sensitivity of the dipole antenna impedances in terms of the measured S-parameters . . . . . . . . . . . . . . 42

5.1.2 The sensitivity of the spiral antenna impedances in terms of the measured S-parameters . . . . . . . . . . . . . . . . . 42

5.1.3 The sensitivity of the impedances to the semi-rigid cables . . . . . . 43

5.1.4 Height of the DUT above the EMC box . . . . . . . . . . . . . . . 43

5.2 Interpretation of the sensitivity analysis over a parameter range . . . . . 43

5.2.1 Sensitivities for the S-parameters and the semi-rigid cables . . . . . 43

5.2 .2 The periodicity of the sensitivities . . . . . . . . . . . . 43

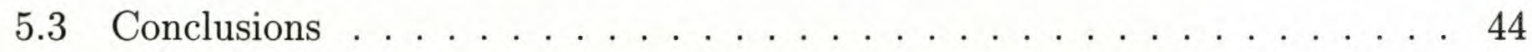

6 Final results $\quad 45$

6.1 Simulation in $\mathrm{FEKO} \ldots \ldots \ldots \ldots \ldots$. . . . . . . . . . . . . . . . . . . . . . .

6.2 The Wu-King profile . . . . . . . . . . . . . . . . 45

6.3 The dipole antenna . . . . . . . . . . . . . . . . . . 46

6.4 The spiral antenna . . . . . . . . . . . . . . . . . . . . . 48

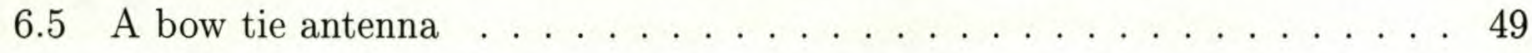

6.6 Sensitivities using the modified jig . . . . . . . . . . . . . 51

6.6.1 Sensitivities for the measured S-parameters and semi-rigid cables . . 51

6.6.2 Stray inductance at the connection point with the DUT . . . . . . 51

7 Conclusions $\quad 55$

$\begin{array}{ll}\text { Appendix } & 58\end{array}$ 


\section{List of Figures}

2.1 Two port impedance model of an antenna and feed. . . . . . . . . . . . 13

2.2 Dipole under measurement using a conventional two port system. . . . . . 14

2.3 Proposed two port measurement jig using a two port network analyzer. . . 15

$3.1 \alpha$ of the semi rigid cables without time gating $\ldots \ldots \ldots$

$3.2 \alpha$ of the semi rigid cables with time gating . . . . . . . . . 17

3.3 Archimedes Spiral Antenna . . . . . . . . . . . . . . 18

3.4 Two different measurements of the magnitude of S11 of the spiral . . . . 18

3.5 Picture of the measurement jig consisting of the semi-rigid cables and the EMC box. . . . . . . . . . . . . . . . . . . . . . 19

3.6 Geometry of the spiral in IE3D . . . . . . . . . . . . . . . . 19

3.7 Comparison between the measured S-parameters of the antenna using the measurement jig, vs. the extracted S-parameters of the antenna alone . . . 20

3.8 Measured vs. simulated results for the differential input impedance of the Archimedes spiral . . . . . . . . . . . . . . . . 21

3.9 The input resistance of the spiral according to IE3D: in the presence or absence of a dielectric . . . . . . . . . . . . . . . 22

3.10 Measured vs. simulated results for the common mode input impedance of the Archimedes spiral . . . . . . . . . . . . . . . . . . . . 22

3.11 Modeling the dipole, EMC box and semi-rigid cables in FEKO . . . . . . . 23

3.12 Measured and simulated differential impedance of the dipole . . . . . . . . 24

3.13 Measured and simulated common mode impedance of the dipole . . . . . . 24

3.14 Enlarged view of the feed section of the dipole in FEKO exciting the antenna in common mode . . . . . . . . . . . . . . . . . . 25

3.15 Comparison of the common mode impedance of the dipole using two feed methods. . . . . . . . . . . . . . . . . . . 2

4.1 Sensitivity of the dipole of $\left|Z_{\text {diff }}\right|$ and $\left|Z_{\text {common }}\right|$ to $|\mathrm{S} 11|,|\mathrm{S} 21|,|\mathrm{S} 12|$ and

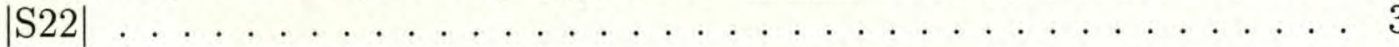


4.2 Sensitivity of the dipole of the angle of $Z_{\text {diff }}$ and the angle of $Z_{\text {common }}$ to

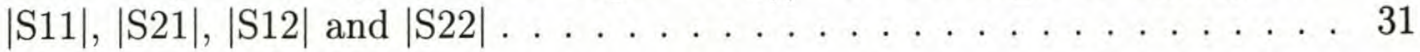

4.3 Sensitivity of $Z_{\text {diff }}$ and $Z_{\text {common }}$ of the dipole to all the S-parameters together 32

4.4 Combined sensitivity of $Z_{\text {diff }}$ and $Z_{\text {common }}$ of the spiral antenna to all the S-parameters

4.5 Sensitivity of $Z_{\text {diff }}$ and $Z_{\text {common }}$ of the dipole antenna for the semi-rigid cables

4.6 Sensitivity of $Z_{\text {diff }}$ and $Z_{\text {common }}$ of the spiral antenna for the semi-rigid cables 35

4.7 The impedance of the dipole for different heights above the EMC box . . . 36

4.8 Comparison of the common mode impedance of the spiral when the stray reactance caused by the feeds are subtracted from the measurements . . . 38

4.9 Comparison between $\left|S 11_{\text {all }}\right|$ and $\left|S 11_{\text {all }}\right|^{2}+\left|S 21_{\text {all }}\right|^{2}$ as measured for the

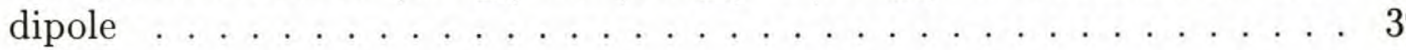

4.10 Sensitivity of $\left|Z_{\text {diff }}\right|$ and $\left|Z_{\text {common }}\right|$ for generally chosen S-parameters using the measurement jig

4.11 Sensitivity of the angle of $Z_{\text {diff }}$ and $Z_{\text {common }}$ for generally chosen S-parameters 41

6.1 Resistance required along the semi-rigid cable to stop the current in FEKO

6.2 Comparison of the differential and common mode impedance of the dipole as measured with a short, standard pair of coaxial cables where only the orientations of the cables were changed . . . . . . . . . .

6.3 Comparison of the differential and common mode impedance of the dipole as measured with a longer pair of coaxial cables where only the orientations of the cables were changed. . . . . . . . . . . . . .

6.4 Comparison of the differential input impedance of the dipole as measured using two methods and simulated in FEKO. . . . . . . . . . . . . . . . .

6.5 Measured vs. simulated result using the short semi-rigid cables and the coaxial cables with higher transfer impedance. . . . . . . . . . . . 49

6.6 The bow tie antenna . . . . . . . . . . . . . . . 50

6.7 The two methods used to measure the bow tie antenna . . . . . . . . . . 50

6.8 Differential input impedance of the bow tie antenna using different measuring techniques ....................... 5

6.9 Sensitivity of $\left|Z_{\text {diff }}\right|$ and $\left|Z_{\text {common }}\right|$ of the spiral for the measured S-parameters using the modified jig . . . . . . . . . . . . . . . . 52

6.10 Sensitivity of $Z_{\text {diff }}$ and $Z_{\text {common }}$ of the spiral for $\alpha$ and $\beta$ of the new semirigid cables

6.11 Measured spiral common mode input impedance compared to that from which the stray inductance at the feed was subtracted 
1 Circuit model for a complex antenna load when both ports of the network analyzer are connected. . . . . . . . . . . . . . . 58 


\section{Chapter 1}

\section{Introduction}

\subsection{Overview}

A problem often encountered in measuring balanced loads such as dipole antennas or some types of microwave amplifiers is that a balun is needed to match the unbalanced line (e.g. a coaxial cable) to the balanced load (e.g. the antenna). This thesis offers a solution to such problems by providing a system in which loads can be measured without the use of a balun. The technique uses the normal two port calibration of the network analyzer, which is extended by the addition of two short lengths of cable. From the measured data, a circuit model of the antenna impedances can be found readily, and both the balanced and unbalanced impedances can be extracted.

\subsection{Current performance limitations of baluns}

In order to properly measure a balanced load, the balun should provide a suitable match between the balanced and unbalanced load over the required bandwidth. Specifically, it must excite the balanced load with a well balanced current. The performance of a balun is determined by factors such as :

1. The bandwidth.

2. The level of unbalance or common mode current relative to the total balanced current in the frequency band of interest.

3. The ratio between the impedances of the balanced and unbalanced loads.

Some antennas, e.g. biconical antennas, may operate over a few decades. The higher the required bandwidth, the greater the difficulty in making a suitable balun. Since baluns are impedance transformers, it becomes increasingly difficult to match two loads of different impedance as the bandwidth increases. Subsequently, a balun affects the accuracy of impedance measurements of the balanced load under test. Currently, a balun 
that demonstrates a very wide operating bandwidth is the improved $C P W_{G F P}$-CPS double $\mathrm{Y}$ balun [1]. It has a frequency bandwidth of a few decades.

\subsection{Research to date in eliminating the need for a balun}

The only literature that could be found was a technique using non-standard calibrations [2] whereby the differential impedance can be obtained. The frequency limitations of the calibration standards limit the bandwidth over which the technique can be applied. 


\section{Chapter 2}

\section{The proposed measurement system}

\subsection{The proposed impedance model of an antenna}

In order to accurately model the impedance of an antenna, provision must be made for a differential and common mode impedance. An ordinary dipole antenna was considered and the following assumptions regarding the impedances were made :

1. The differential impedance can be modelled by a complex impedance between the dipole arms.

2. The common mode impedance can be modelled by a complex impedance between each dipole arm and ground.

The proposed model is shown in fig 2.1. Vd and Vc are the balanced and unbalanced excitation voltages, nodes $\mathrm{A}$ and $\mathrm{B}$ are the antenna feed terminals and $\mathrm{Za}, \mathrm{Zb}$ (normally $\mathrm{Za}=\mathrm{Zb}$ ) and $\mathrm{Zc}$ model the antenna impedances. The impedances, $\mathrm{Za}$ and $\mathrm{Zb}$, are the impedances between each antenna terminal and ground, and $\mathrm{Zc}$ is the impedance between the antenna terminals. The common mode impedance is $Z_{\text {common }}=Z a \| Z b$ and the differential mode input impedance is $Z_{\text {diff }}=Z c \|(Z a+Z b)$ (see appendix for derivation). The antenna or balanced load under test, is modelled as a 3 terminal system. If, for example, a dipole is considered, the one arm of the dipole can be assumed to be connected to terminal A and the other arm of the dipole on terminal B. The signal ground in the case of the dipole may be the ground of the transmission lines feeding the dipole arms. In order to visualize this more clearly, figure 2.2 shows the dipole connected in a conventional two port network. On the left and right of the figure are the ports of the network analyzer. Each arm of the dipole is connected to one port of the network analyzer. The dipole impedances are also shown with the modified connections indicated by dashed lines.

\section{$2.2 \quad$ The physical model}

The proposed physical layout for measuring balanced loads is shown in fig. 2.3. Here a 


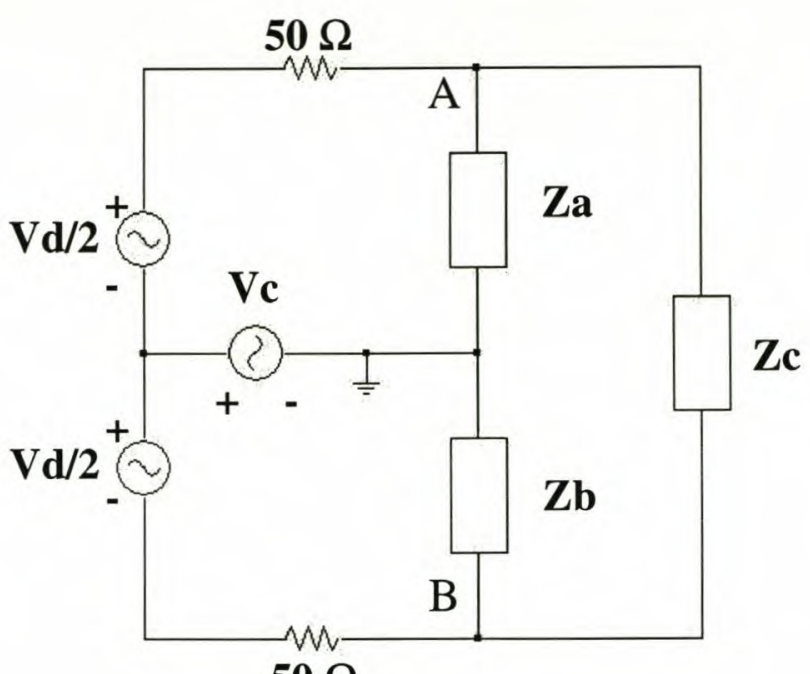

$50 \Omega$

Figure 2.1: Two port impedance model of an antenna and feed.

network analyzer is shown where each port is connected by coaxial cables to one of the semi-rigid cables. The two semi-rigid cables are soldered together on the outer conductors, and are used as port extensions to bring the feed points close to the antenna terminals.

\subsection{Measurement procedure}

The procedure for measuring the antenna is as follows :

1. The network analyzer is calibrated at the connection ports using the manufacturer supplied standards.

2. The parameters of the two semi-rigid cables are found from a short circuit measurement.

3. The antenna S-parameters are obtained by de-embedding the influence of the semi rigid cables from the S-parameters measured at the SMA connectors.

4. The antenna impedances are derived from the S-parameters.

\subsection{Removing the effect of the semi rigid cables}

As an approximation, the semi-rigid cable is modelled as an attenuation, $\alpha$, and a phase delay, $\beta$. A short to ground is placed on the end of the semi rigid cables and the complex, 


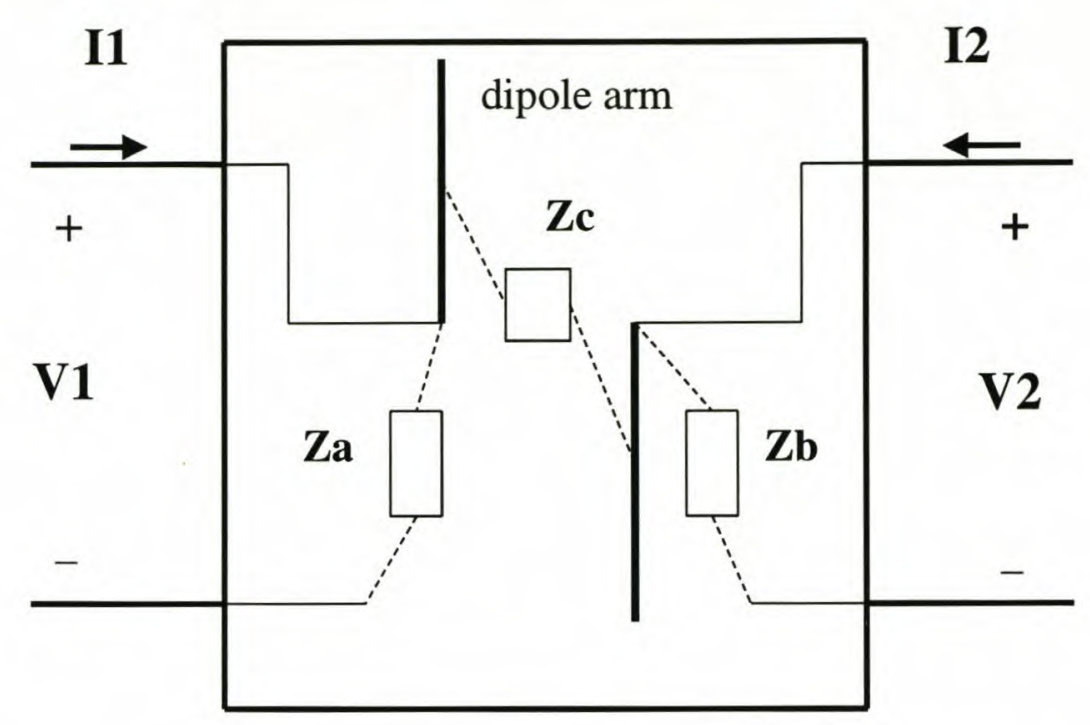

Figure 2.2: Dipole under measurement using a conventional two port system.

input reflection coefficients of both cables are measured. The phase of the measurement yields $\beta l$ as the measured phase represents twice the electrical length of the cable as well as a 180 degree phase reversal due to the short placed at the ends.

The attenuation over the length of the line is obtained by dividing the magnitude (in Nepers) of the reflection coefficient by 2 to yield $\alpha l$. With $\alpha$ and $\beta$ of the two semi-rigid cables known, their ABCD parameters may each be written as follows [3] :

$$
\left[\begin{array}{ll}
A & B \\
C & D
\end{array}\right]=\left(\begin{array}{cc}
\cosh (\alpha+j \beta) l & Z_{0} \sinh (\alpha+j \beta) l \\
\frac{1}{Z_{0}} \sinh (\alpha+j \beta) l & \cosh (\alpha+j \beta) l
\end{array}\right)
$$

where $Z_{0}$ was taken as unity for the normalised case.

It must be mentioned that the model assumes no reflections from the connections at the SMA connectors. Since they were typically lower than $-20 \mathrm{~dB}$, such reflections were neglected.

The S-parameters of the full measurement are converted to ABCD-parameters through [4]. These values are then pre-multiplied by the inverse matrix of the ABCD-parameters of the semi rigid cable on port 1 and post-multiplied by the inverse matrix of the ABCDparameters of the semi rigid cable on port 2. The extracted ABCD-parameters of the antenna are then converted to Y-parameters. The Y-parameters, when de-normalised to $50 \Omega$, yield the required impedances as shown in the appendix. 
dipole antenna

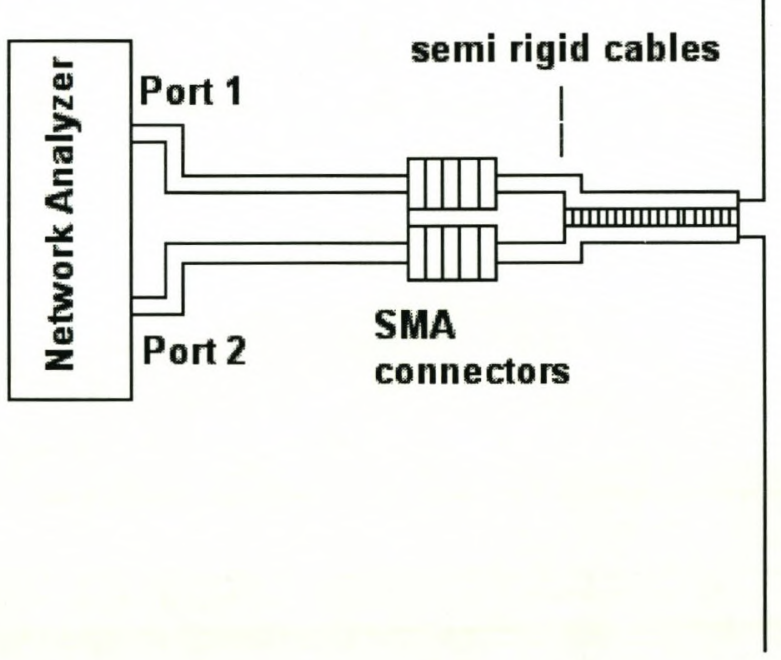

Figure 2.3: Proposed two port measurement jig using a two port network analyzer. 


\section{Chapter 3}

\section{Initial measured and simulated results}

In this chapter results are presented for a spiral antenna and for a dipole.

\subsection{The semi-rigid cables}

The semi-rigid cables were shorted at the ends by making an electrical connection between the centre conductors and the outer conductors using aluminum foil. The reflection coefficients were subsequently measured. Initial measurements revealed that the short placed at the end of the cables requires special attention in that a short made by using aluminum foil was not as accurate as using solder. This was particularly evident in the reflection coefficient. The magnitude of the reflection coefficient was lower for the aluminum foil compared to when the electrical connection was established by soldering the inner and outer conductors together. In addition, using more solder seemed to increase the magnitude even further. This implied that the short was able to radiate some of the input power if it was not perfect, in effect behaving like a small, badly matched antenna. Additional solder was applied until the magnitude of the reflection coefficient stopped increasing.

Figure 3.1 shows $\alpha$ for one of the semi-rigid cables. The curve for $\alpha$ displayed periodic behaviour with respect to frequency. This was probably the result of reflections inside the semi-rigid cable between the short and the connection with the SMA connector. Using the HP8510 network analyzer's time gating function, the true value of $\alpha$ could be found by eliminating all but the main reflected pulse in the semi-rigid cables. This was necessary since the model of the semi-rigid cables was a basic representation consisting of only a loss and phase parameter. The value of $\beta$ remained constant with or without the use of time-domain gating. Figure 3.2 shows $\alpha$ with time domain gating.

\subsection{Spiral antenna}

The subject of the first measurement was the spiral antenna shown in figure 3.3 with parameters as follows: 


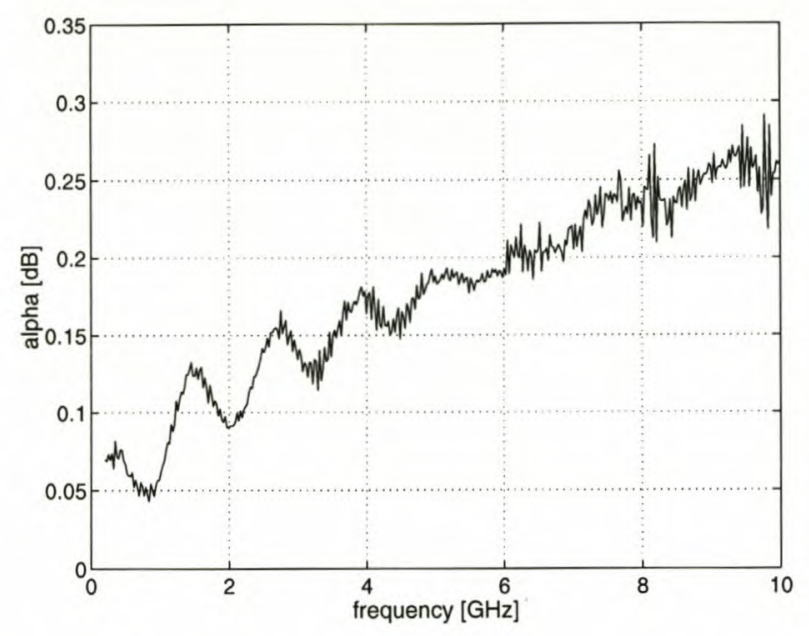

Figure 3.1: $\alpha$ of the semi rigid cables without time gating

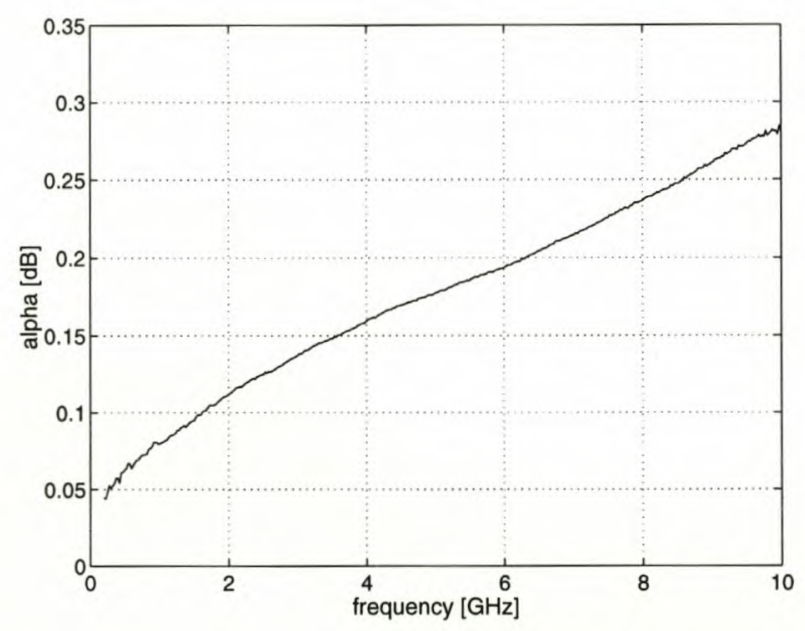

Figure 3.2: $\alpha$ of the semi rigid cables with time gating

Polarization: right circular ;

Number of revolutions: 6.5 ;

Antenna diameter: inner $5 \mathrm{~mm}$, outer $90 \mathrm{~mm}$;

Substrate: $0.55 \mathrm{~mm}, \epsilon_{r}=3$.

The centre conductors of the semi-rigid cables were each connected to one of the arms of the spiral. A length of approximately $20 \mathrm{~cm}$ was preferred for the semi-rigid cables. During the initial measurement procedure, the magnitude of the measured S-parameters was seen to change as a result of electrical contact being made between the connectors of the semirigid cables. Figure 3.4 shows a difference of up to $1 \mathrm{~dB}$ between the measurements with and without contact between the connectors. It was postulated that the problem was due to the common mode currents flowing on the outside of the outer conductors of the semi-rigid cables. 


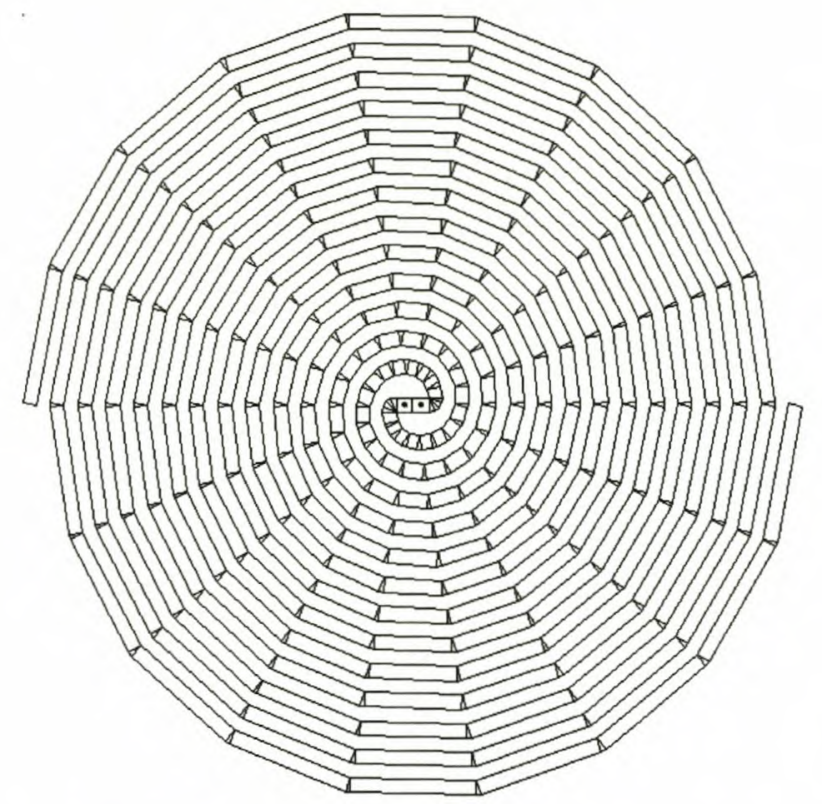

Figure 3.3: Archimedes Spiral Antenna

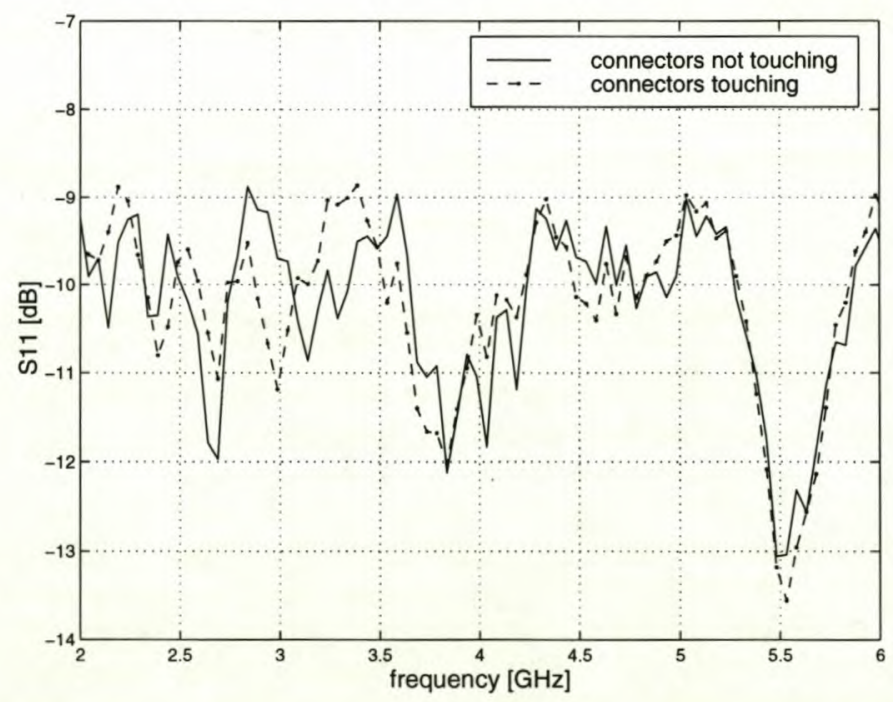

Figure 3.4: Two different measurements of the magnitude of S11 of the spiral 


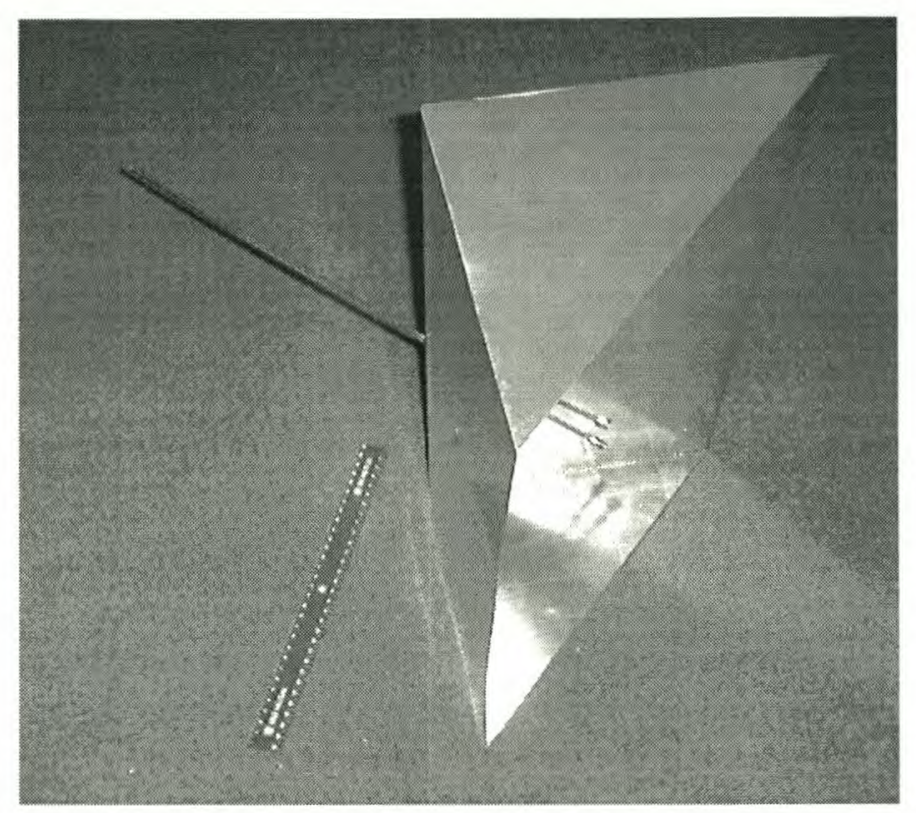

Figure 3.5: Picture of the measurement jig consisting of the semi-rigid cables and the EMC box.

In order to reduce this effect, an EMC box was added to the measurement apparatus. This consisted of a ground plane of roughly $50 \mathrm{~cm}^{2}$ bent in a triangular shape. To allow the semi-rigid cables to pass through the ground plane, a small hole was drilled in the centre of the box. Figure 3.5 shows a picture of the semi-rigid cables and the EMC box.

A numerical tool, IE3D [5], was used to simulate the spiral. The model for the spiral in IE3D is shown in figure 3.6.

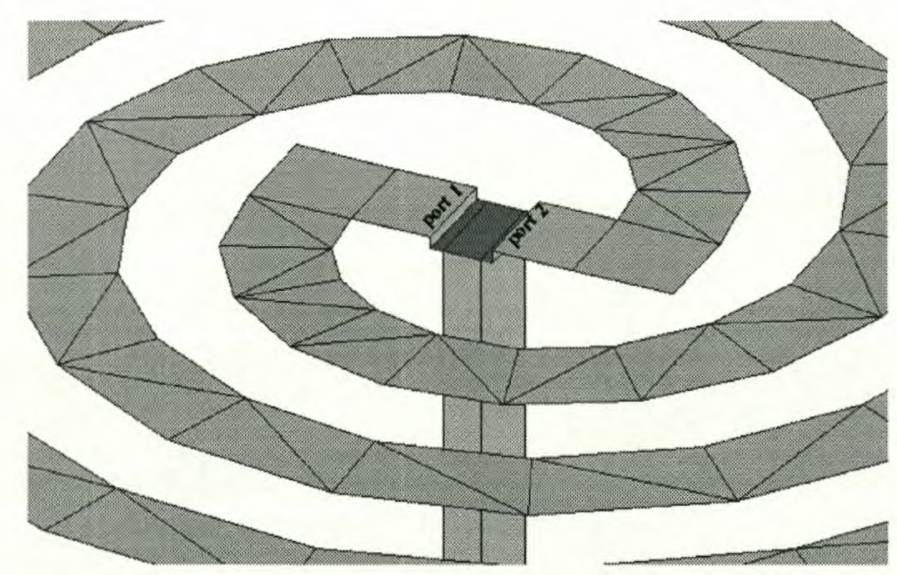

Figure 3.6: Geometry of the spiral in IE3D

A vertical offset was added at the spiral feed point so that a vertically localised port could be used. The semi-rigid cables were modelled as a single rectangular cylinder, $40 \mathrm{~cm}$ long, connected to a ground plane. Due to limitations in IE3D's representation of three dimensional objects, the exact geometry of the EMC box could not be implemented. Instead, the structure was modelled as a flat ground plane with the same surface area as the EMC box. 
With these EMC issues taken into account, the measurements were repeated. When the connectors of the semi-rigid cables made contact, only a slight difference (approximately $0.05 \mathrm{~dB}$ ) was measured in the S-parameters. This was deemed a satisfactory reduction in the common mode current flow on the semi rigid cables.

Fig 3.7 shows how the S-parameters of the antenna compare to those of the full measurement. The curves for the full measurement show the measured S-parameters of the

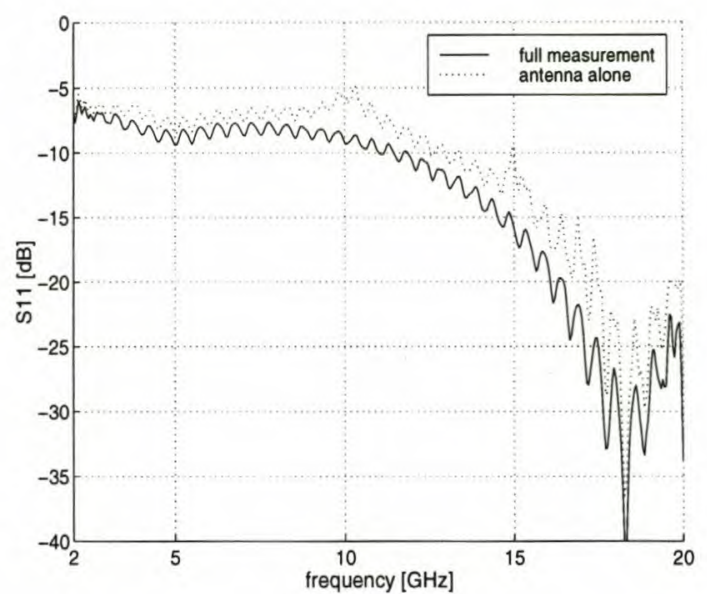

(a) $|\mathrm{S} 11|$

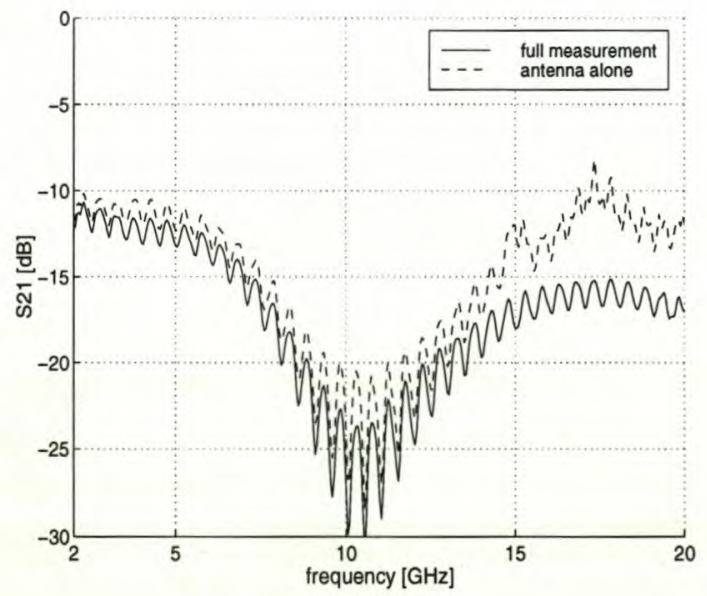

(c) $|\mathrm{S} 21|$

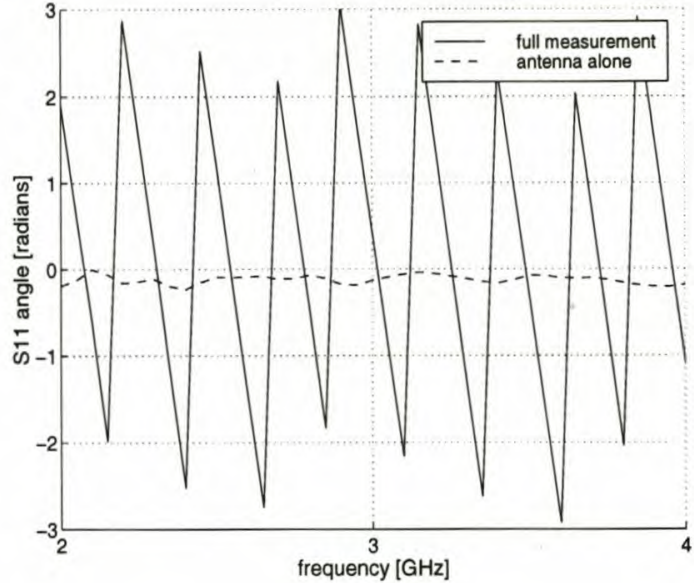

(b) angle S11

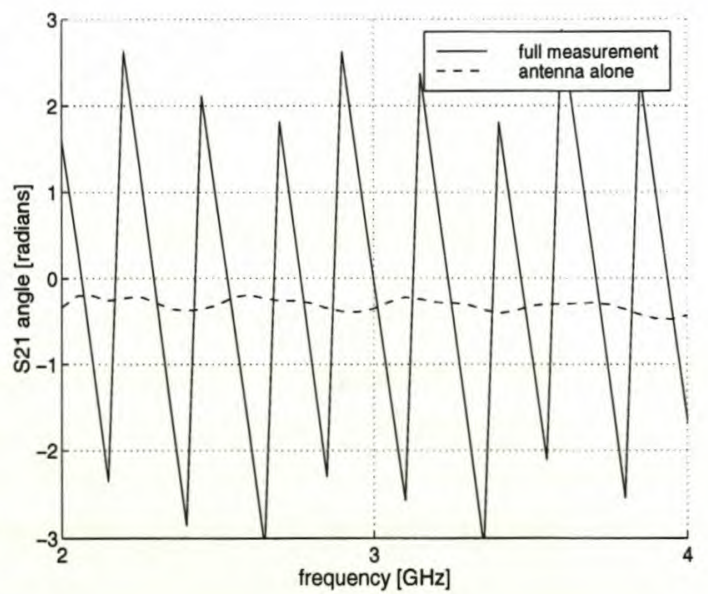

(d) angle S21

Figure 3.7: Comparison between the measured S-parameters of the antenna using the measurement jig, vs. the extracted S-parameters of the antenna alone

antenna including the effects of the semi-rigid cables. The curves for the antenna alone show the S-parameters of the antenna alone, i.e. where the effect of the semi-rigid cables has been extracted from the S-parameters of the full measurement. The distinction between the two sets of measurements demonstrates the effects of the semi-rigid cables and the necessity to properly extract their effect. 
Figure 3.8 shows the measured and simulated results for the differential input impedance of the Archimedes spiral antenna supported by a dielectric. Three curves are shown for

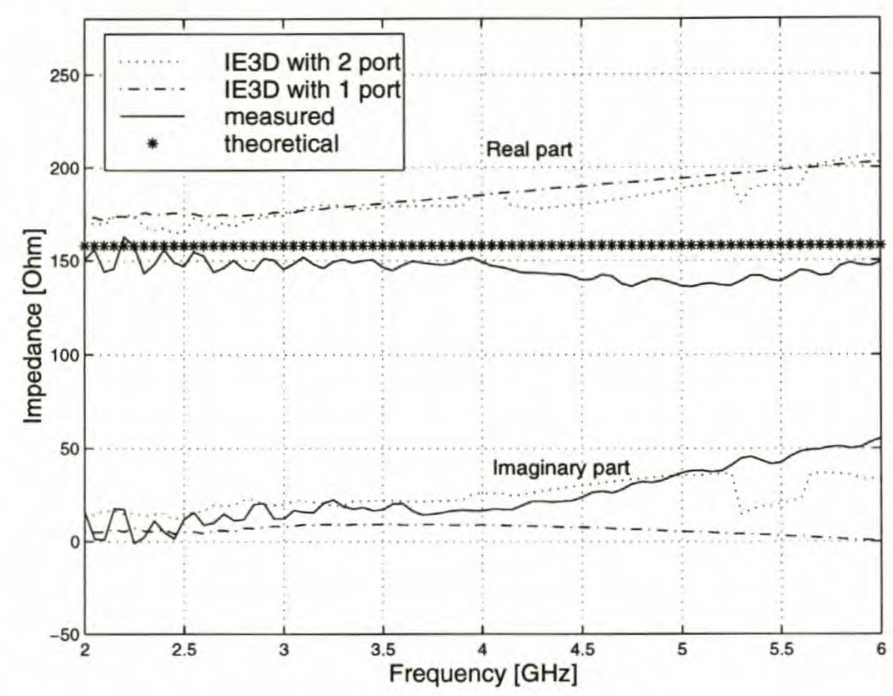

Figure 3.8: Measured vs. simulated results for the differential input impedance of the Archimedes spiral

both the real and imaginary parts of the impedance as well as a curve depicting the theoretical value. One of the pairs of curves shows the antenna being fed by a two port in IE3D, approximating the proposed technique for measuring the antenna. Each of the centre conductors of the semi-rigid cables are connected to their own separate antenna terminals. Another pair of curves shows the antenna in IE3D being fed differentially by only one port. (This represents the conventional method whereby the antenna is fed using a balun). The remaining pair shows the results of the proposed measurement technique. The measured and simulated results agree well in both form and value. The spiral in free space has a theoretical value of $188 \Omega$ due to its self complementary characteristic, however an average of $150 \Omega$ has been measured. This difference can be attributed to the dielectric layer supporting the spiral. An IE3D simulation (using the 1 port method) for verification with and without the dielectric showed that the dielectric drops the impedance by an average of $30 \Omega$ implying an expected impedance value of $158 \Omega$. This is in good agreement with the measured average of $150 \Omega$. See fig.3.9.

The common mode impedance is shown in figure 3.10. Though not certain, the disagreements between the measurements and simulation results are probably due to the absence of the dielectric layer as was the case with the differential impedance. In addition, differences in reflections from the flat ground plane in simulation vs. reflections in measurement from the triangular shaped EMC box can not be disregarded. 


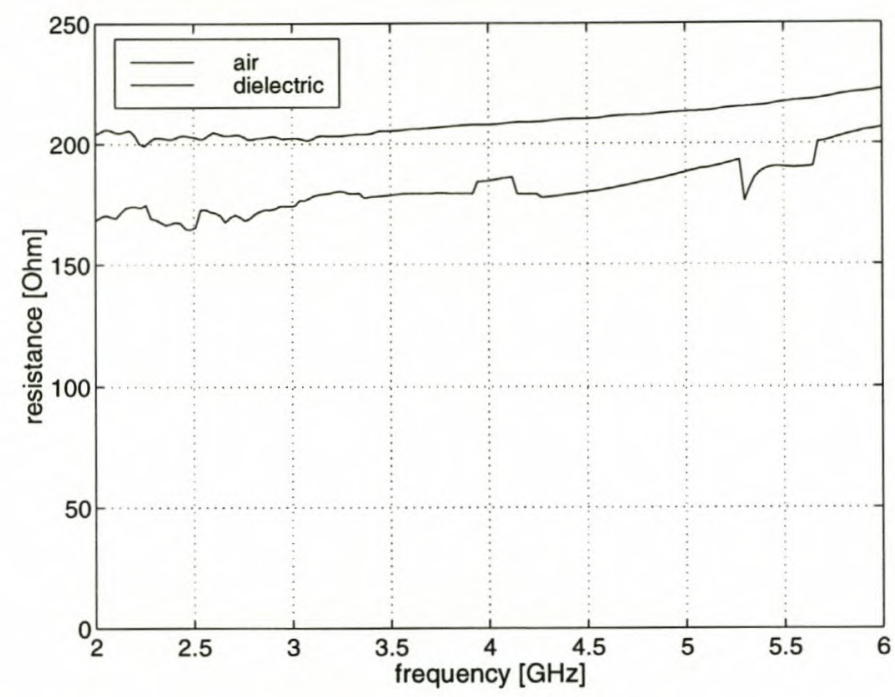

Figure 3.9: The input resistance of the spiral according to IE3D: in the presence or absence of a dielectric

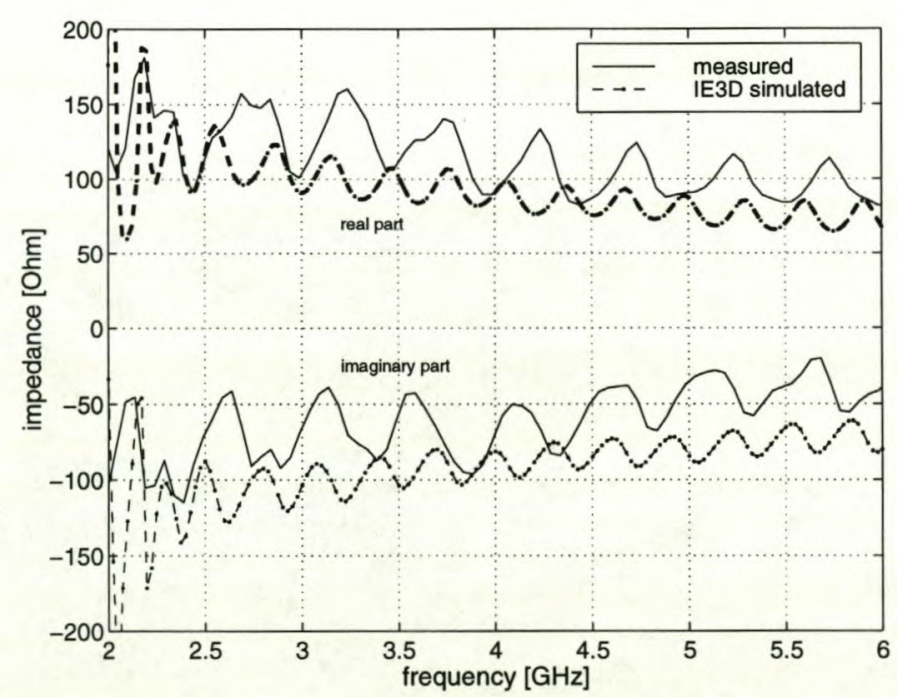

Figure 3.10: Measured vs. simulated results for the common mode input impedance of the Archimedes spiral 


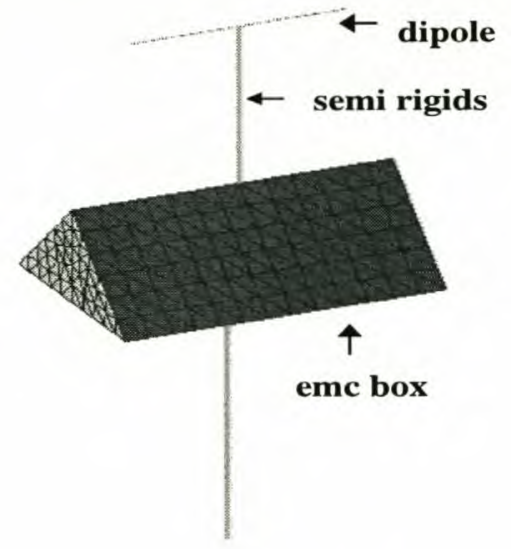

(a) complete model

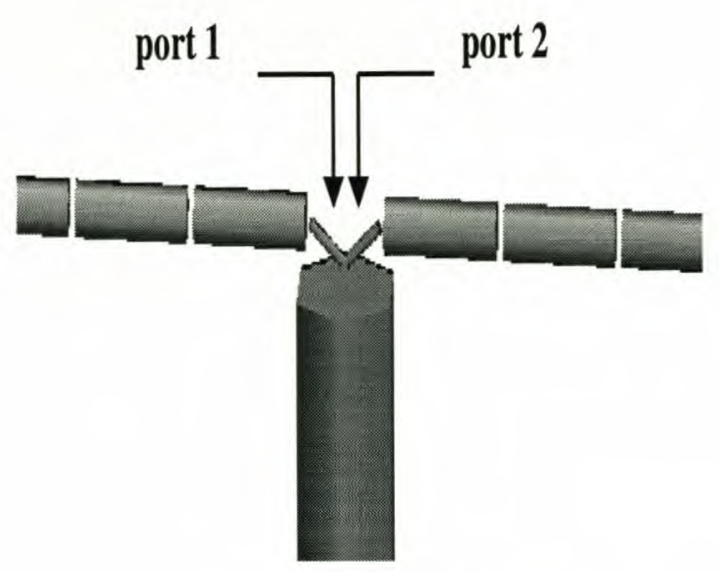

(b) enlarged view of feed

Figure 3.11: Modeling the dipole, EMC box and semi-rigid cables in FEKO

\subsection{Dipole antenna}

For the next measurement, a test dipole was constructed by soldering two pieces of brazing rod, each $152 \mathrm{~mm}$ long and $3 \mathrm{~mm}$ thick, onto the centre conductors of the semi-rigid cables. The dipole, the semi-rigid cables and the EMC box were modelled using the numerical tool, FEKO [6]. The semi-rigid cables were modelled by a thick wire of approximately the same radius as the two semi-rigid cables combined. The EMC box was modelled as is. Figure 3.11 shows the full model in FEKO as well as an enlarged view, depicting the feed of the dipole in the simulated two-port measurement. Fig. 3.12 depicts the measured vs. simulated results for the input impedance, $Z_{\text {diff }}$. As with the spiral in fig. 3.8 , three curves are shown. The curve for the one port simulation is nearly indistinguishable from those of the two port simulations. Also of interest is the common mode input impedance of the dipole. Fig. 3.13 depicts the real and imaginary parts of the common mode impedance, $Z_{\text {common. }}$ For the differential impedance, the agreement at the lower frequencies is good, but deteriorates somewhat at the higher frequencies. The agreement is not as good in the case of the common mode impedance as it is for the differential impedance.

\subsubsection{Confirmation of the common mode impedance model}

In order to validate the antenna impedance model of fig. 2.1 with regards to the common mode impedance, the dipole antenna was simulated again using only one port. In simulation, the two dipole arms were joined together at the feed point and a delta gap feed was inserted between the cylinder (representing the semi-rigid cables) and the feed point (see fig. 3.14). Fig. 3.15 compares the common mode impedance in FEKO using the two feeding methods. The good agreement between the curves show that both modelling methods are accurate. 


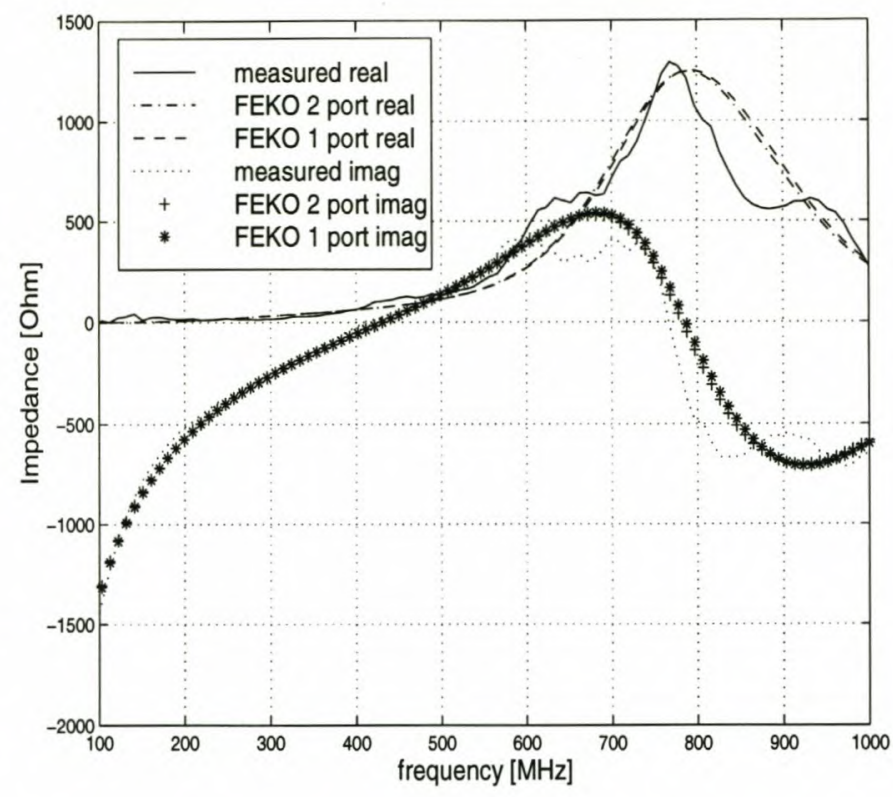

Figure 3.12: Measured and simulated differential impedance of the dipole

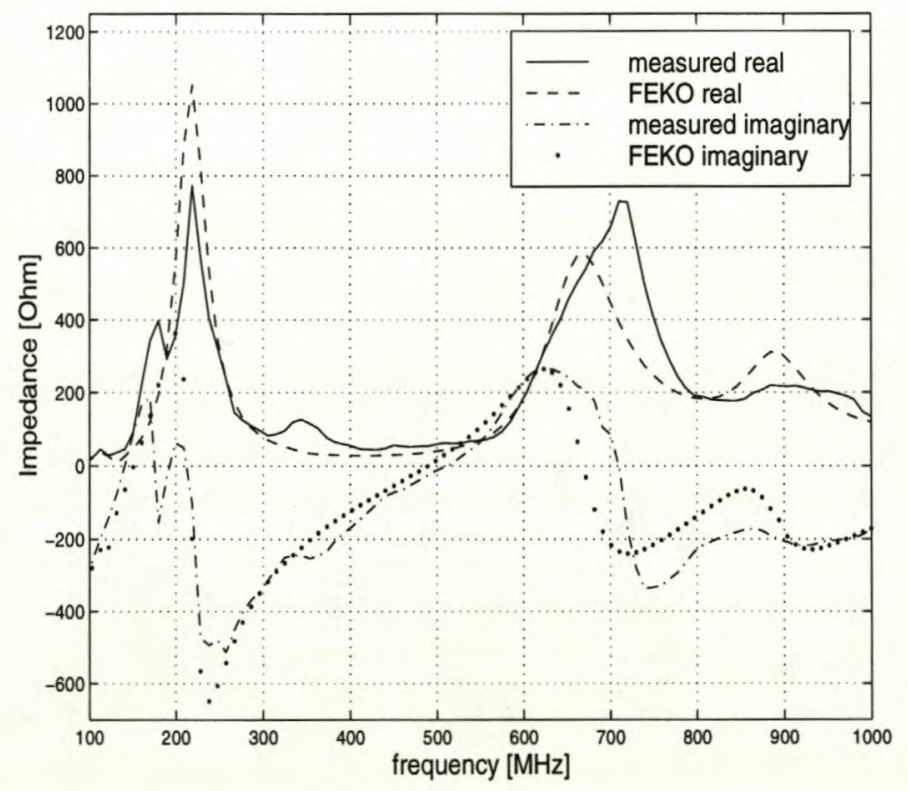

Figure 3.13: Measured and simulated common mode impedance of the dipole 


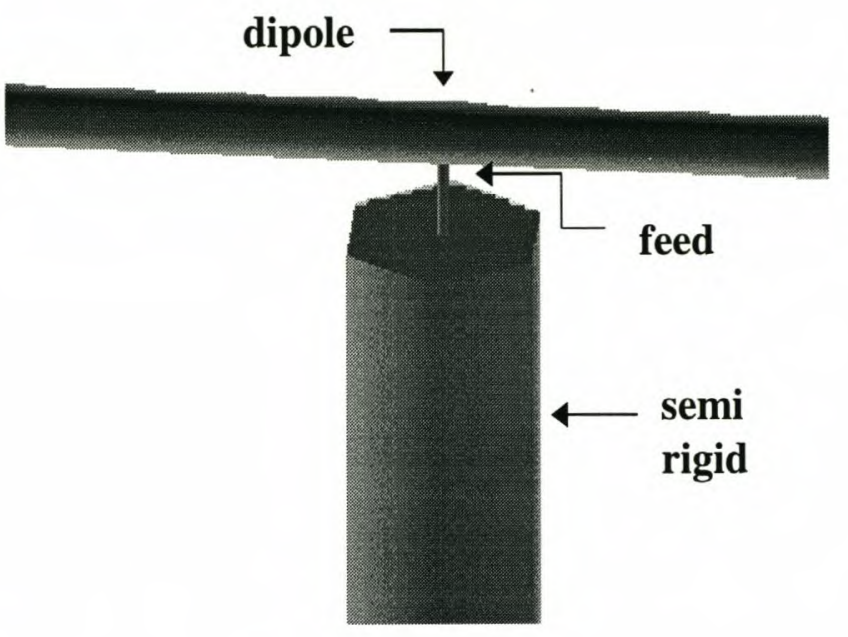

Figure 3.14: Enlarged view of the feed section of the dipole in FEKO exciting the antenna in common mode

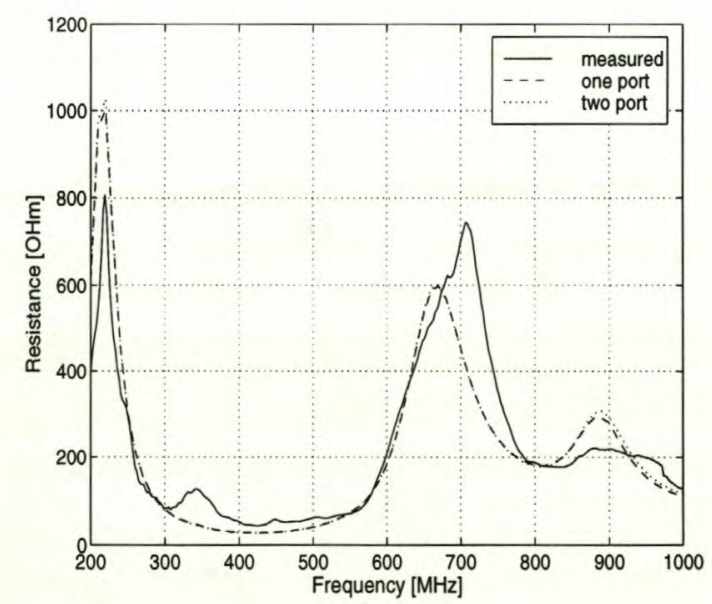

(a) real part

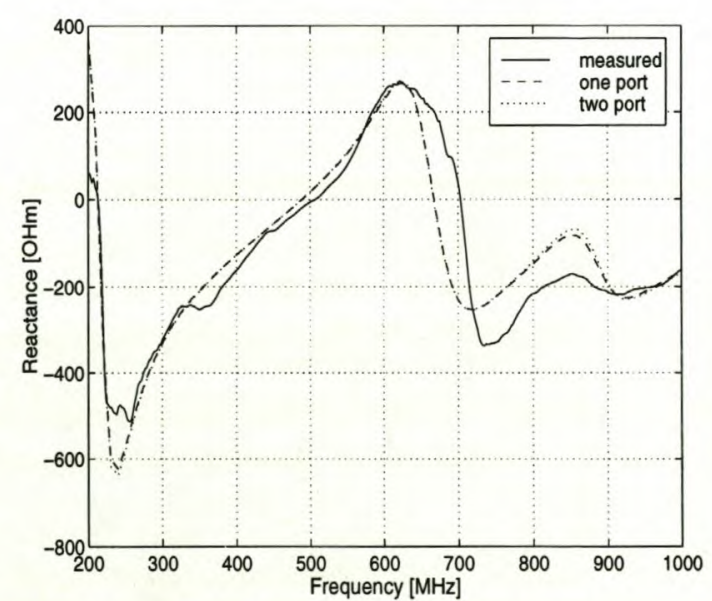

(b) imaginary part

Figure 3.15: Comparison of the common mode impedance of the dipole using two feed methods. 


\section{Chapter 4}

\section{Sensitivity analysis}

In order to identify the cause of the discrepancies between the measurements and the simulations of chapter 3 , a sensitivity analysis was necessary to determine which variables in the measurement procedure have the greatest influence on the results. The variables of interest are :

1. The semi-rigid cables

2. The role of the EMC box as a reflecting ground plane

3. The S-parameters of the full measurement

4. Stray inductance at the connection point with the antenna

In this chapter the sensitivity results are presented, but the reader is referred to chapter 5 for their interpretation.

\subsection{The sensitivity equation}

The sensitivity of some performance measure, $y$, with respect to an element value, $x$, is given by [7]

$$
S_{x}^{y}=\frac{x}{y} \cdot \frac{d y}{d x}
$$

If $\mathrm{y}$ is a function of several variables $\left[y=f\left(x 1, x 2, \ldots, x_{n}\right)\right]$, then the sensitivity of $\mathrm{y}$ with respect to $x_{i}$ is

$$
S_{x_{i}}^{y}=\frac{x}{y} \cdot \frac{\partial y}{\partial x} \quad \mathrm{i}=1,2, \ldots, \mathrm{n}
$$

The sensitivity, $\mathrm{S}$, is given as a percentage. The definition given in 4.2 is known as the differential, first-order, classical, relative, or Bode sensitivity. Since sensitivities with respect to both the magnitude and the phase were necessary for several variables, a complex sensitivity analysis was performed. Since (4.2) does not provide information on complex values, the sensitivity equation may readily be derived for a complex network function, 
$H=|H| e^{j \phi}$, as follows:

$$
\begin{aligned}
S_{x}^{H} & =\frac{x}{H} \cdot \frac{\partial H}{\partial x} \\
& =\frac{x}{|H| e^{j \phi}} \cdot\left[\frac{\partial|H|}{\partial x} \cdot e^{j \phi}+j|H| e^{j \phi} \cdot \frac{\partial \phi}{\partial x}\right] \\
& =\frac{\partial|H|}{\partial x} \cdot \frac{x}{|H|}+j \frac{\partial \phi}{\partial x} \cdot x \\
& =S_{x}^{|H|}+j U S_{x}^{\phi}
\end{aligned}
$$

where $U S_{x}^{\phi}$ is the unnormalised sensitivity of the phase of $\mathrm{H}$ with respect to $\mathrm{x}$.

\subsection{Obtaining the sensitivity equation for the antenna impedances in terms of the measured S-parameters.}

The sensitivity of the differential and common mode impedances to errors in the Sparamaters of the full measurement was determined. In order to confirm the correctness of the sensitivities, an analysis similar to a Monte Carlo analysis was also performed. I.e. a $1 \%$ error in the S-paramaters of the full measurement was introduced on purpose to compare the impedances with error to those without error. The sensitivity equation (4.2) required the expression of the differential and common mode impedances in terms of the measured S-parameters. The measured S-parameters, $S 11_{\text {all }}, S 21_{\text {all }}, S 12_{\text {all }}, S 22_{\text {all }}$ are known. The differential and common mode impedances, $Z_{\text {diff }}$ and $Z_{\text {common }}$, of the antenna are required. These were derived as follows. Firstly the ABCD-parameters of the full measurement were found using [4]:

$$
\begin{aligned}
A_{\text {all }} & =\frac{\left(1+S 11_{\text {all }}\right)\left(1-S 22_{\text {all }}\right)+S 12_{\text {all }} S 21_{\text {all }}}{2 S 21_{\text {all }}} \\
B_{\text {all }} & =50 \frac{\left(1+S 11_{\text {all }}\right)\left(1+S 22_{\text {all }}\right)-S 12_{\text {all }} S 21_{\text {all }}}{2 S 21_{\text {all }}} \\
C_{\text {all }} & =\frac{\left(1-S 11_{\text {all }}\right)\left(1-S 22_{\text {all }}\right)-S 12_{\text {all }} S 21_{\text {all }}}{100 S 21_{\text {all }}} \\
D_{\text {all }} & =\frac{\left(1-S 11_{\text {all }}\right)\left(1+S 22_{\text {all }}\right)+S 12_{\text {all }} S 21_{\text {all }}}{2 S 21_{\text {all }}}
\end{aligned}
$$

where $A_{\text {all }}, B_{\text {all }}, C_{\text {all }}$ and $D_{\text {all }}$ are the ABCD-parameters of the full measurement. Next the ABCD-parameters of the stand-alone antenna were found. These were obtained by extracting the influence of the semi-rigid cables from the measurement. As stated earlier, this required the pre- and post multiplication of the ABCD-parameters of the full measurement with the inverse of the ABCD-parameters of the male and female semi-rigid cable respectively. The ABCD-parameters of the antenna alone were then obtained as : 


$$
\begin{aligned}
A_{\text {ant }}= & \left(A_{\text {all }} \cosh \gamma l_{m}-50 C_{\text {all }} \sinh \gamma l_{m}\right) \cosh \gamma l_{f} \\
& -0.02\left(B_{\text {all }} \cosh \gamma l_{m}-50 D_{\text {all }} \sinh \gamma l_{m}\right) \sinh \gamma l_{f} \\
B_{\text {ant }}= & -50\left(A_{\text {all }} \cosh \gamma l_{m}-50 C_{\text {all }} \sinh \gamma l_{m}\right) \sinh \gamma l_{f} \\
& +\left(B_{\text {all }} \cosh \gamma l_{m}-50 D_{\text {all }} \sinh \gamma l_{m}\right) \cosh \gamma l_{f} \\
C_{\text {ant }}= & \left(-0.02 A_{\text {all }} \sinh \gamma l_{m}+C_{\text {all }} \cosh \gamma l_{m}\right) \cosh \gamma l_{f} \\
& -0.02\left(-0.02 B_{\text {all }} \sinh \gamma l_{m}+D_{\text {all }} \cosh \gamma l_{m}\right) \sinh \gamma l_{f} \\
D_{\text {ant }}= & -50\left(-0.02 A_{\text {all }} \sinh \gamma l_{m}+C_{\text {all }} \cosh \gamma l_{m}\right) \sinh \gamma l_{f} \\
& +\left(-0.02 B_{\text {all }} \sinh \gamma l_{m}+D_{\text {all }} \cosh \gamma l_{m}\right) \cosh \gamma l_{f}
\end{aligned}
$$

where $\gamma l_{m}$ and $\gamma l_{f}$ represent $\alpha+j \beta$ (see 2.1) of the male and female semi-rigid cable respectively. Next, the Y-parameters of the antenna were found, also using [4] :

$$
\begin{aligned}
Y 11_{a n t} & =\frac{D_{a n t}}{B_{a n t}} \\
Y 12_{a n t} & =\frac{B_{a n t} C_{a n t}-A_{a n t} D_{a n t}}{B_{a n t}} \\
Y 21_{a n t} & =\frac{-1}{B_{a n t}} \\
Y 22_{a n t} & =\frac{A_{\text {ant }}}{B_{\text {ant }}}
\end{aligned}
$$

Using the Y-parameters, the impedances $Z_{a}, Z_{b}$ and $Z_{c}$ could be found (see appendix for derivation).

$$
\begin{aligned}
Z_{\text {a ant }} & =\frac{1}{Y 11_{\text {ant }}+Y 21_{\text {ant }}} \\
Z_{b \text { ant }} & =\frac{1}{Y 22_{a n t}+Y 21_{\text {ant }}} \\
Z_{\text {cant }} & =\frac{-1}{Y 21_{\text {ant }}}
\end{aligned}
$$

where $Z_{a \text { ant }}, Z_{b \text { ant }}$ and $Z_{c \text { ant }}$ are the impedances as shown in fig.2.1. Finally, the impedances $Z_{\text {diff }}$ and $Z_{\text {common }}$ are given by (as also shown in the appendix) :

$$
\begin{aligned}
Z_{\text {diff }} & =\frac{Z_{c}\left(Z_{a}+Z_{b}\right)}{Z_{a}+Z_{b}+Z_{c}} \\
Z_{\text {common }} & =\frac{Z_{a} Z_{b}}{Z_{a}+Z_{b}}
\end{aligned}
$$

The equations mentioned above are sufficient for expressing the antenna impedances, $Z_{\text {diff }}$ and $Z_{\text {common }}$ in terms of the measured S-parameters, thereby enabling the use of equation 4.2 .

\subsection{Sensitivity analysis of the measured data}

In this section the sensitivities for the antennas, semi-rigid cables and general setup in chapter 3 are determined. 


\subsubsection{The sensitivity of the dipole antenna impedances in terms of the measured S-parameters}

The sensitivities of the impedances $Z_{\text {diff }}$ and $Z_{\text {common }}$ were first calculated analytically using 4.2. These sensitivities were confirmed by the Monte Carlo analysis mentioned previously. The results of the analytical method were found using symbolic manipulation by a computer program. Figure 4.1 shows the magnitude sensitivities of $Z_{\text {diff }}$ and $Z_{\text {common }}$ with respect to the magnitude of the measured S-parameters $S 11_{\text {all }}, S 21_{\text {all }}, S 12_{\text {all }}$ and $S 22_{\text {all }}$. Fig. 4.2 shows the angle sensitivities of $Z_{\text {diff }}$ and $Z_{\text {common }}$ with respect to the magnitude of the measured S-parameters. Figure 4.3 shows the sensitivities of $Z_{\text {diff }}$ and $Z_{\text {common }}$ of the dipole for all the S-parameters. This is obtained by adding the moduli of the sensitivities for the individual S-parameters. Unfortunately, due to the fact that the angles of the impedances cross through zero, causing division by zero in the normalization process, the angle sensitivities could not be expressed in their normalized form.

It must be noted that the results shown in figure 4.3 pertain to the specific set of measured S-parameters of the dipole and semi-rigid cables combination.

It is clear that the two methods for determining the sensitivities yield virtually the same results. The slight discrepancy between the two results is a consequence of a first order approximation in the sensitivity equation (4.2). Higher order derivatives need to be taken into account to achieve higher accuracy.

\subsubsection{The sensitivity of the spiral antenna impedances in terms of the measured S-parameters}

Figure 4.4 shows the sensitivities for the spiral antenna impedances with respect to the magnitude of all the S-parameters together. Since the accuracy of both methods for determining the sensitivities has been verified by their good agreement, it was not deemed necessary to show the curves for both methods.

\subsubsection{The sensitivity of the impedances to the semi-rigid cables}

The effect of the semi rigid cables on the results was determined by investigating how sensitive the results were to the semi-rigid cable parameters, $\alpha$ and $\beta$. Figure 4.5 and 4.6 show the results of such a study for the dipole and spiral antenna respectively. 


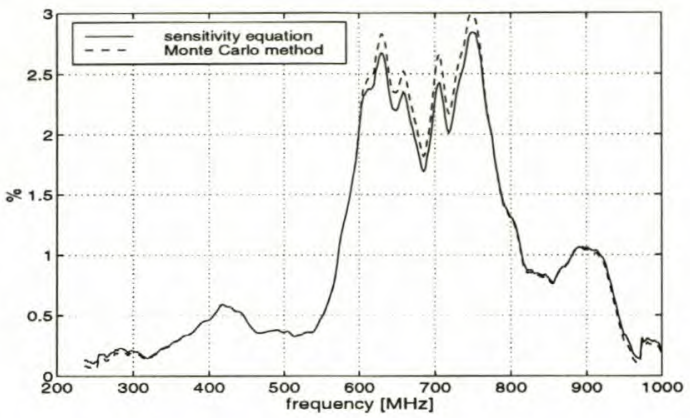

(a) $\left|Z_{\text {diff }}\right|$ to $|\mathrm{S} 11|$

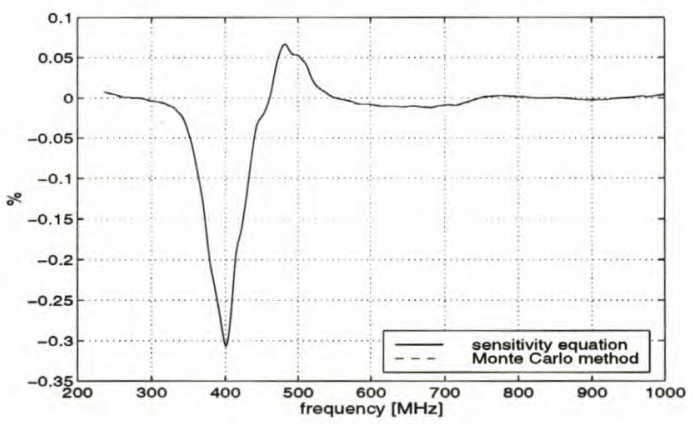

(c) $\left|Z_{\text {diff }}\right|$ to $|\mathrm{S} 12|$

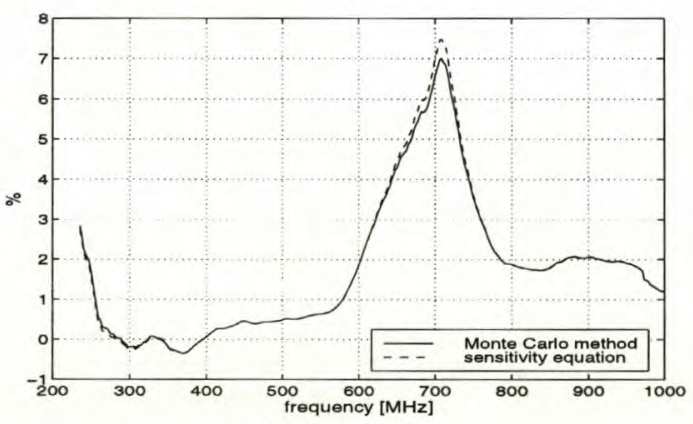

(e) $\left|Z_{\text {common }}\right|$ to $|\mathrm{S} 11|$

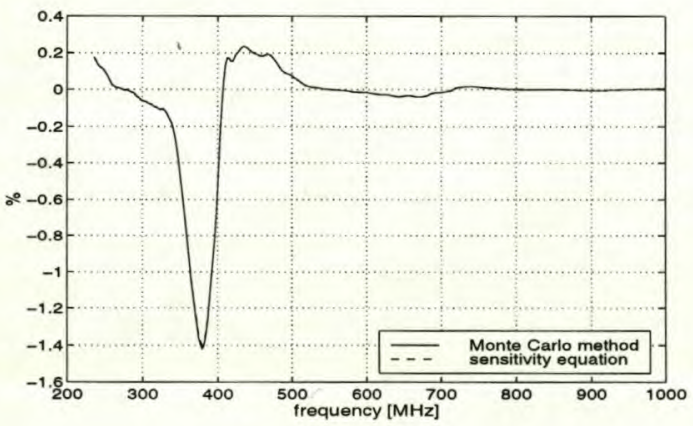

(g) $\left|Z_{\text {common }}\right|$ to $|\mathrm{S} 12|$

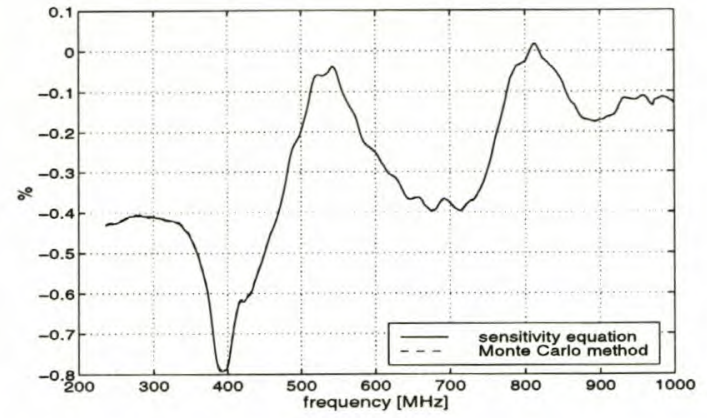

(b) $\left|Z_{\text {diff }}\right|$ to $|\mathrm{S} 21|$

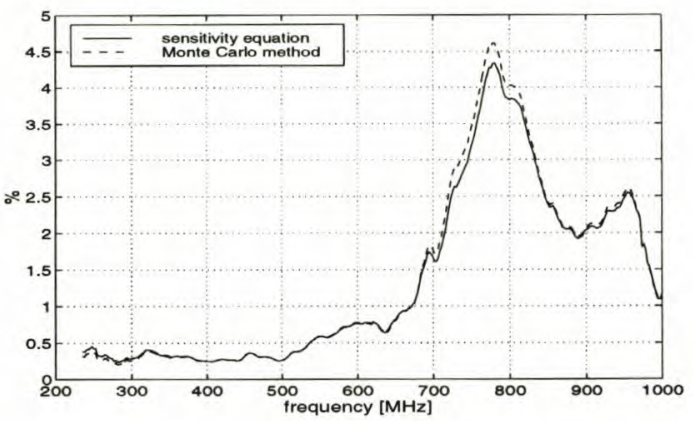

(d) $\left|Z_{\text {diff }}\right|$ to $|\mathrm{S} 22|$

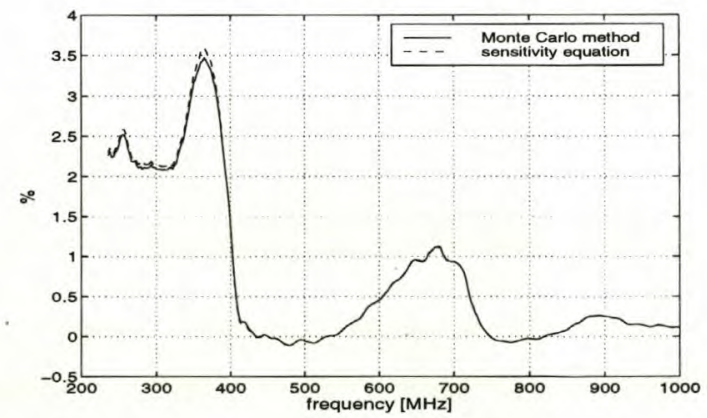

(f) $\left|Z_{\text {common }}\right|$ to $|\mathrm{S} 21|$

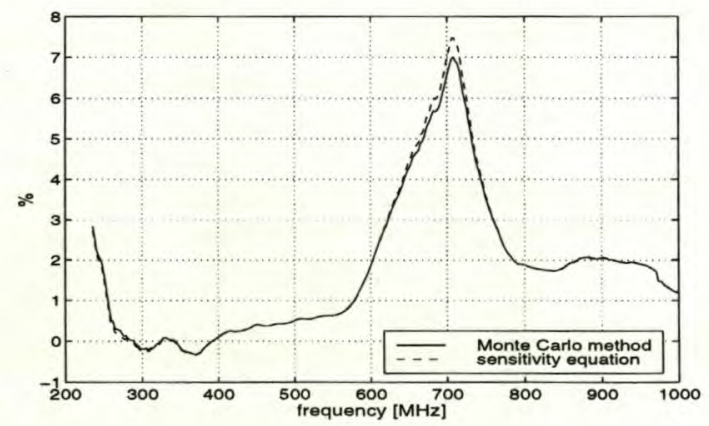

(h) $\left|Z_{\text {common }}\right|$ to $|\mathrm{S} 22|$

Figure 4.1: Sensitivity of the dipole of $\left|Z_{\text {diff }}\right|$ and $\left|Z_{\text {common }}\right|$ to $|\mathrm{S} 11|,|\mathrm{S} 21|,|\mathrm{S} 12|$ and $|\mathrm{S} 22|$ 


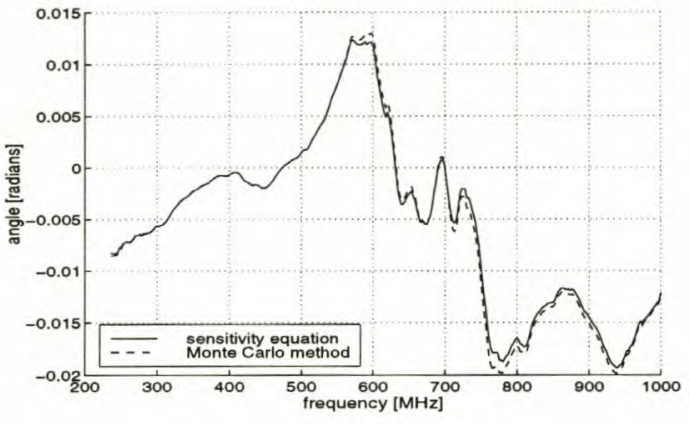

(a) angle $Z_{\text {diff }}$ to $|\mathrm{S} 11|$

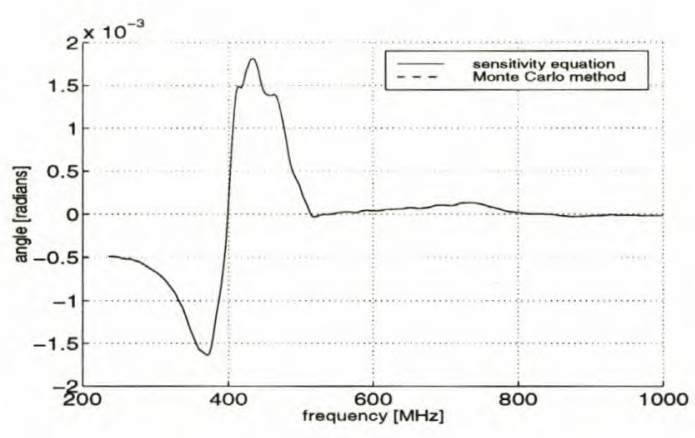

(c) angle $Z_{\text {diff }}$ to $|\mathrm{S} 12|$

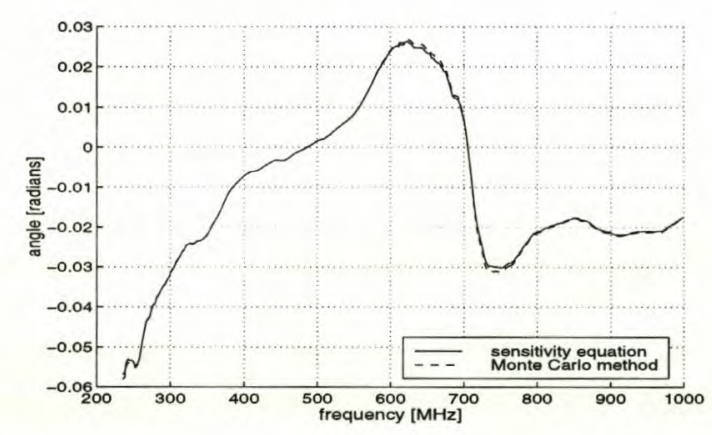

(e) angle $Z_{\text {common }}$ to $|\mathrm{S} 11|$

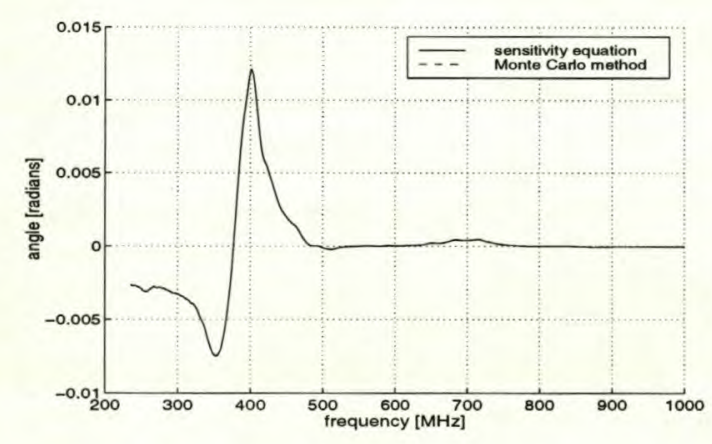

(g) angle $Z_{\text {common }}$ to $|\mathrm{S} 12|$

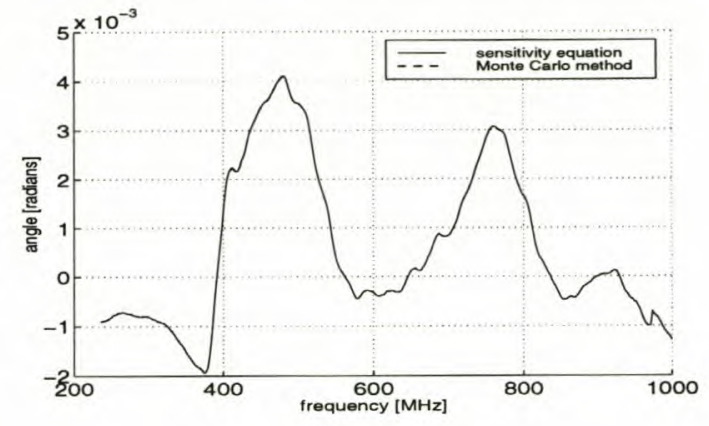

(b) angle $Z_{\text {diff }}$ to $|\mathrm{S} 21|$

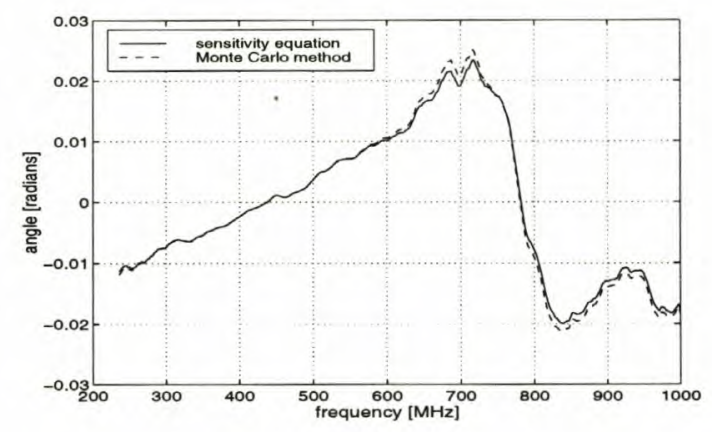

(d) angle $Z_{\text {diff }}$ to $|\mathrm{S} 22|$

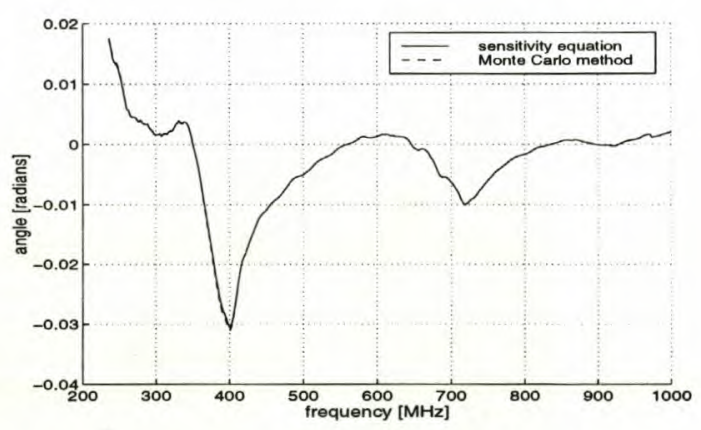

(f) angle $Z_{\text {common }}$ to $|\mathrm{S} 21|$

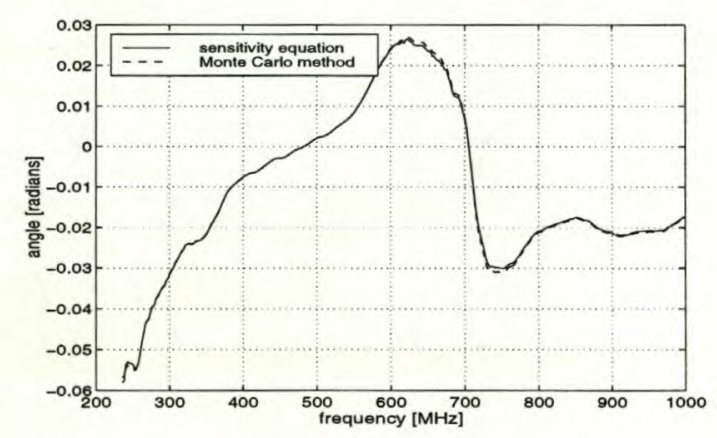

(h) angle $Z_{\text {common }}$ to $|\mathrm{S} 22|$

Figure 4.2: Sensitivity of the dipole of the angle of $Z_{\text {diff }}$ and the angle of $Z_{\text {common }}$ to |S11|, |S21|, |S12| and |S22| 


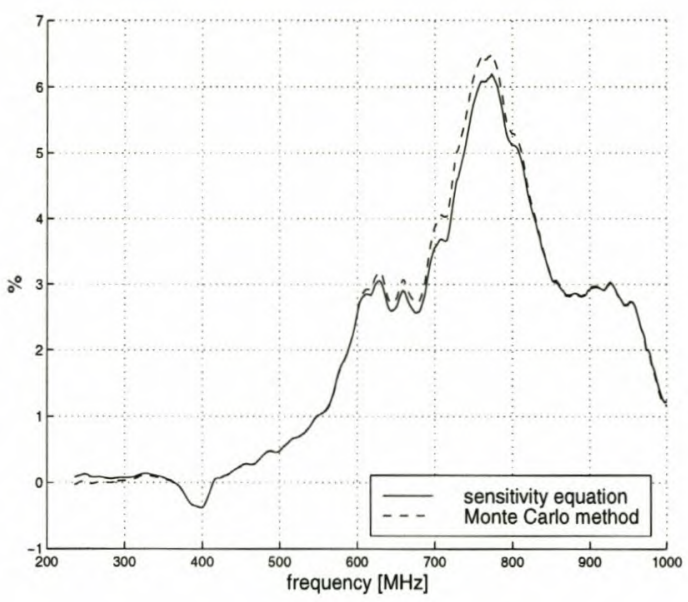

(a) $\left|Z_{\text {diff }}\right|$

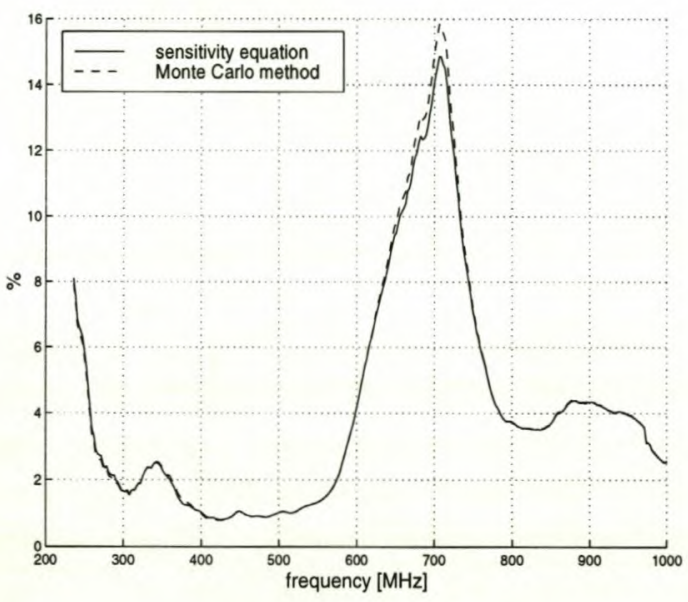

(c) $\left|Z_{\text {common }}\right|$

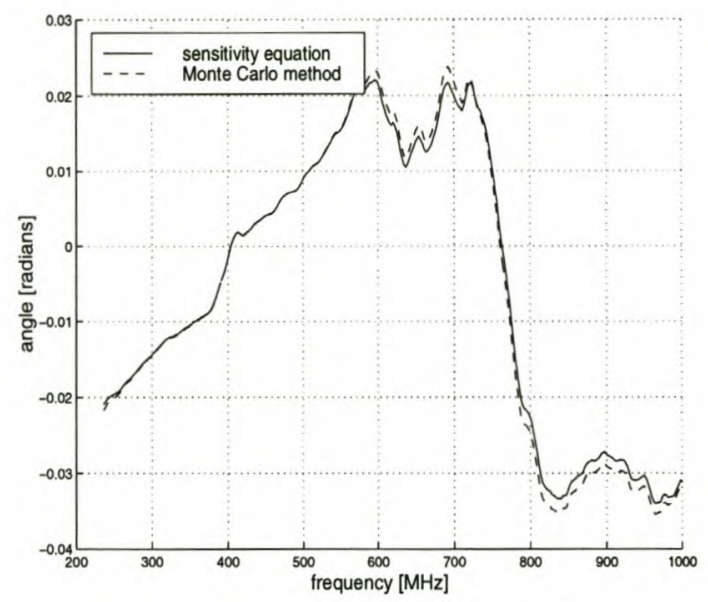

(b) angle $Z_{\text {diff }}$

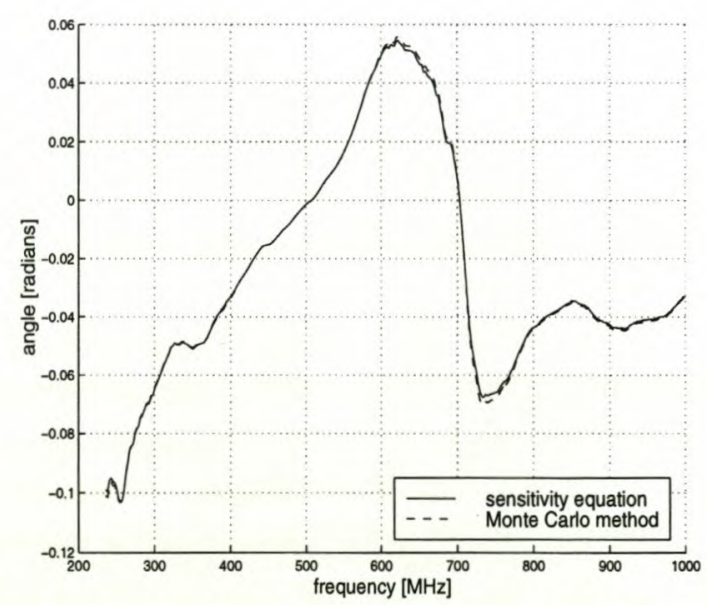

(d) angle $Z_{\text {common }}$

Figure 4.3: Sensitivity of $Z_{\text {diff }}$ and $Z_{\text {common }}$ of the dipole to all the S-parameters together 


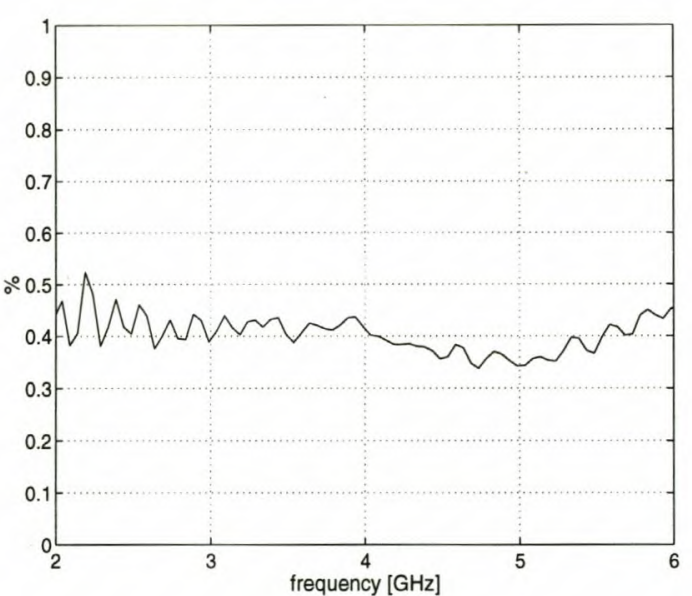

(a) $\left|Z_{\text {diff }}\right|$

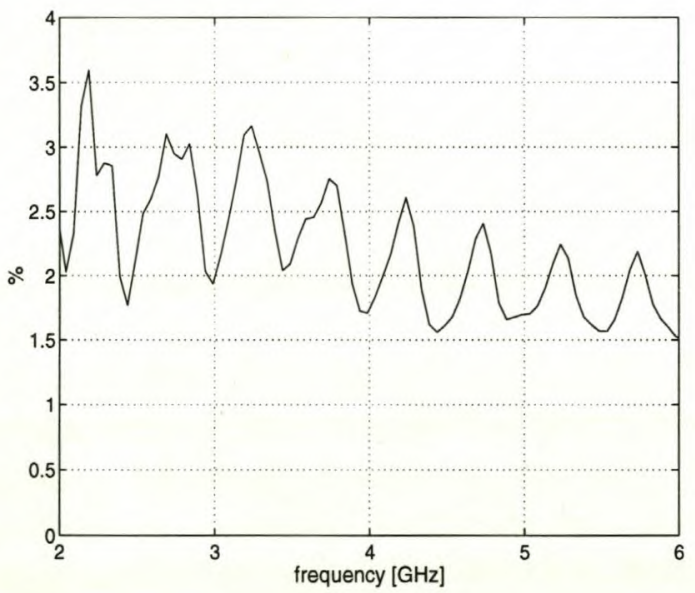

(c) $\left|Z_{\text {common }}\right|$

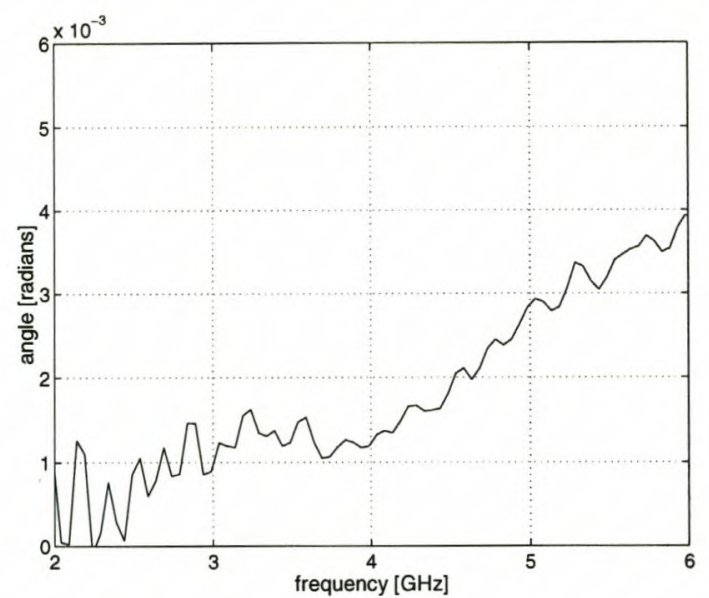

(b) angle $Z_{\text {diff }}$

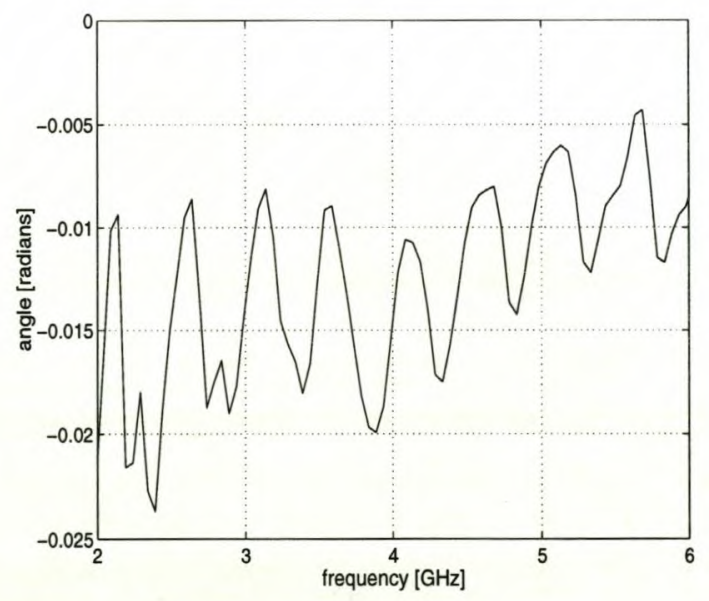

(d) angle $Z_{\text {common }}$

Figure 4.4: Combined sensitivity of $Z_{\text {diff }}$ and $Z_{\text {common }}$ of the spiral antenna to all the S-parameters 


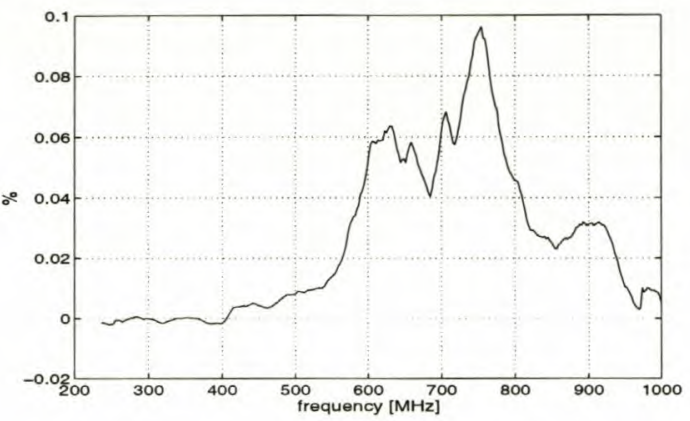

(a) $\left|Z_{\text {diff }}\right|$ for $\alpha$

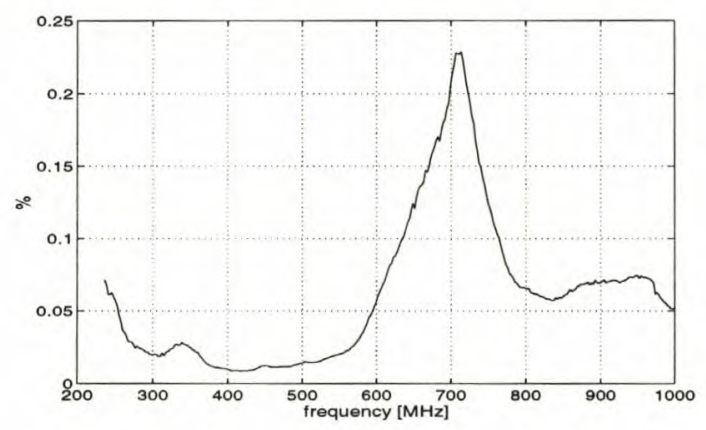

(c) $\left|Z_{\text {common }}\right|$ for $\alpha$

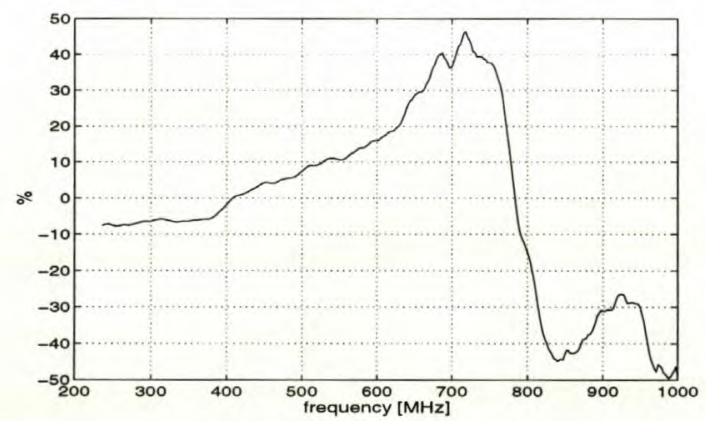

(e) $\left|Z_{\text {diff }}\right|$ for $\beta$

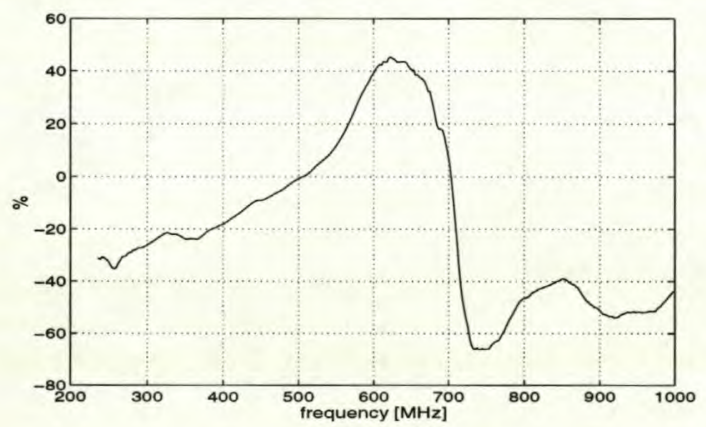

(g) $\left|Z_{\text {common }}\right|$ for $\beta$

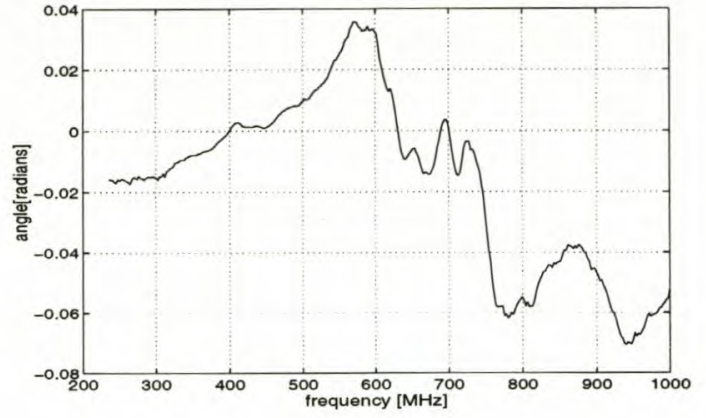

(b) angle $Z_{\text {diff }}$ for $\alpha$

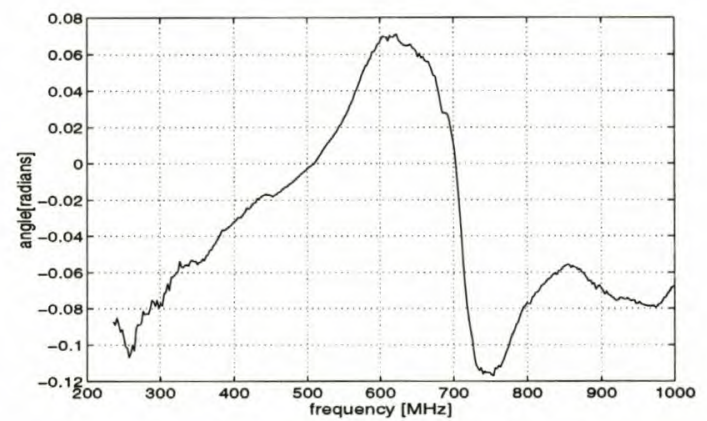

(d) angle $Z_{\text {common }}$ for $\alpha$

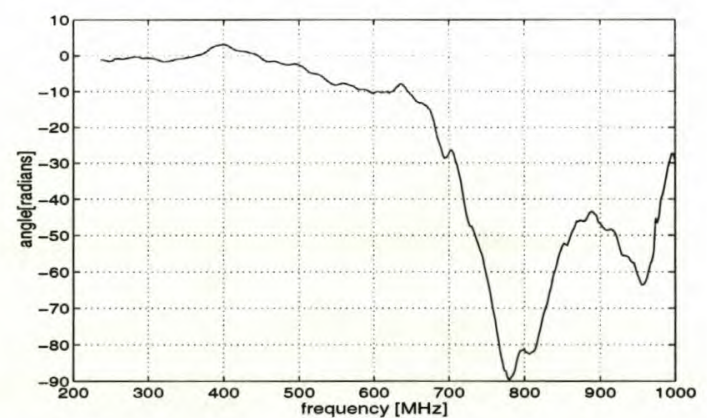

(f) angle $Z_{\text {diff }}$ for $\beta$

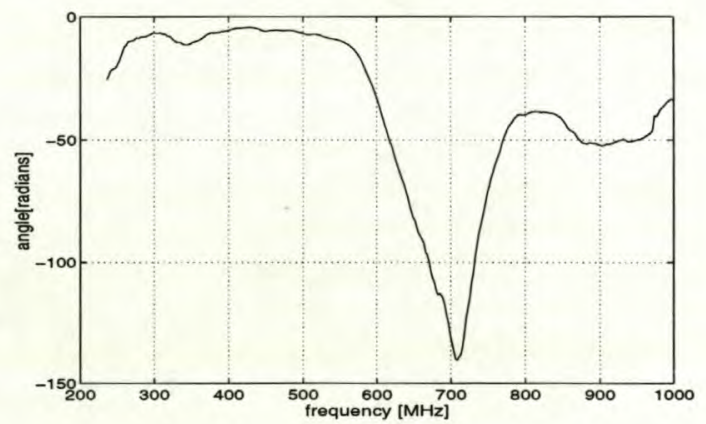

(h) angle $Z_{\text {common }}$ for $\beta$

Figure 4.5: Sensitivity of $Z_{\text {diff }}$ and $Z_{\text {common }}$ of the dipole antenna for the semi-rigid cables 


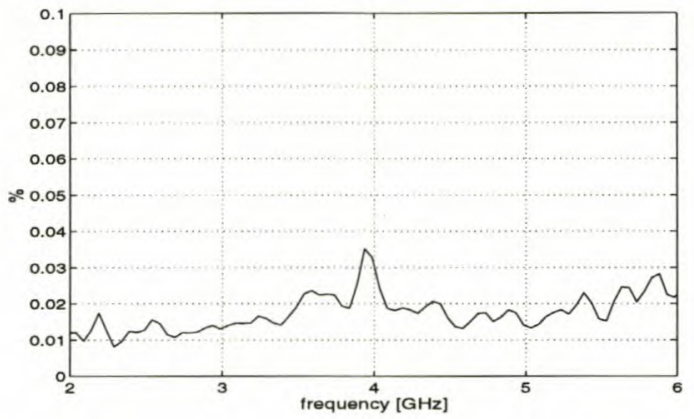

(a) $\left|Z_{\text {diff }}\right|$ for $\alpha$

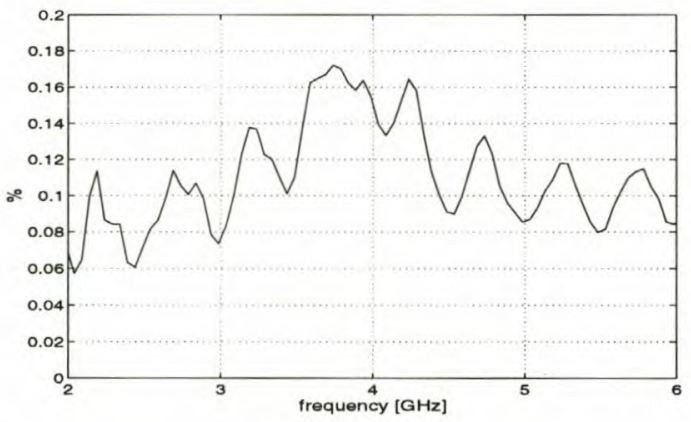

(c) $\left|Z_{\text {common }}\right|$ for $\alpha$

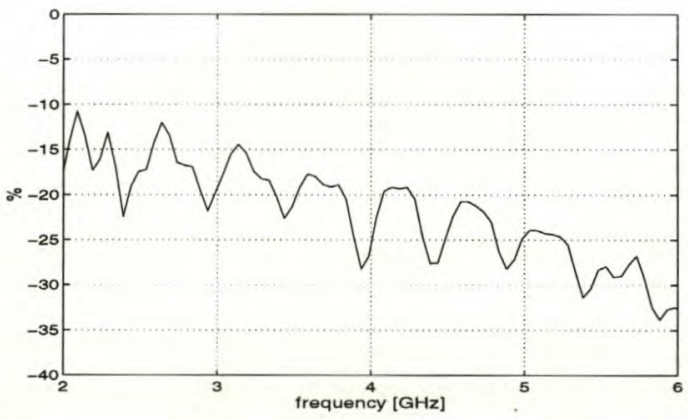

(e) $\left|Z_{\text {diff }}\right|$ for $\beta$

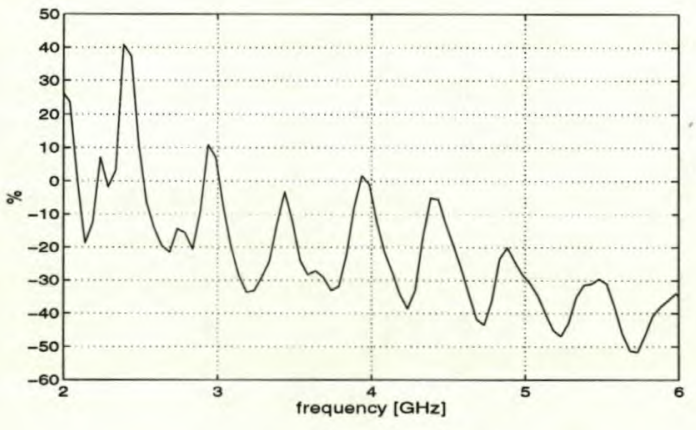

(g) $\left|Z_{\text {common }}\right|$ for $\beta$

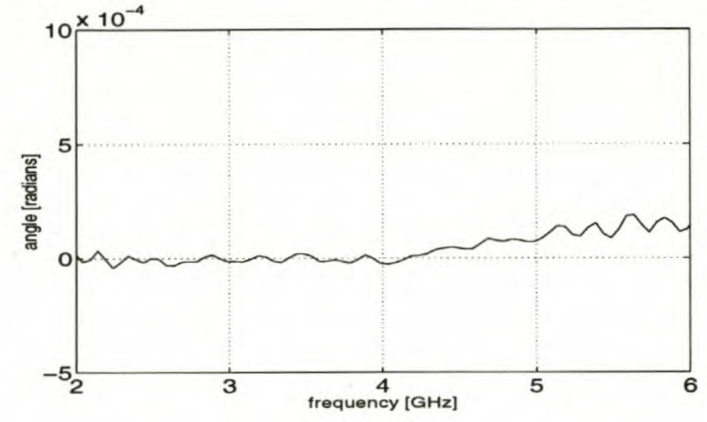

(b) angle $Z_{\text {diff }}$ for $\alpha$

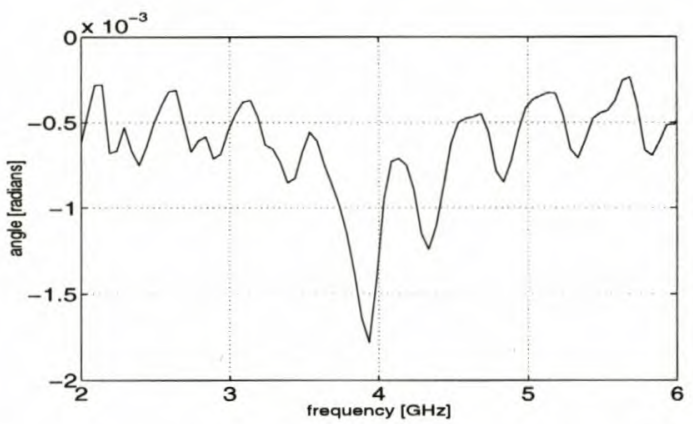

(d) angle $Z_{\text {common }}$ for $\alpha$

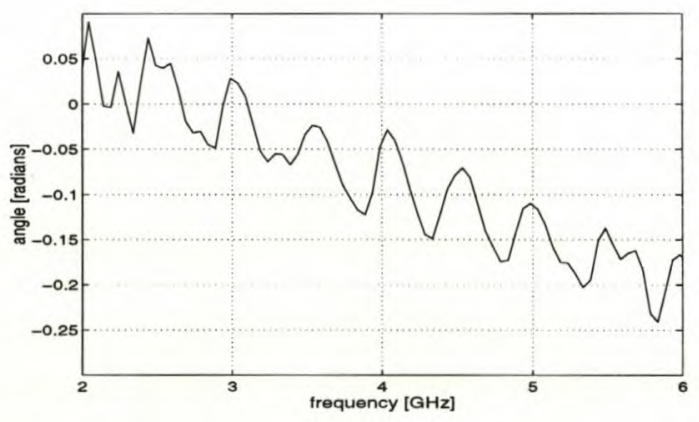

(f) angle $Z_{\text {diff }}$ for $\beta$

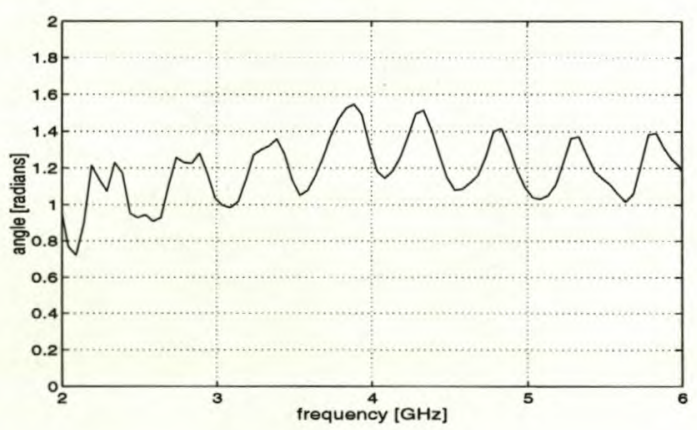

(h) angle $Z_{\text {common }}$ for $\beta$

Figure 4.6: Sensitivity of $Z_{\text {diff }}$ and $Z_{\text {common }}$ of the spiral antenna for the semi-rigid cables 


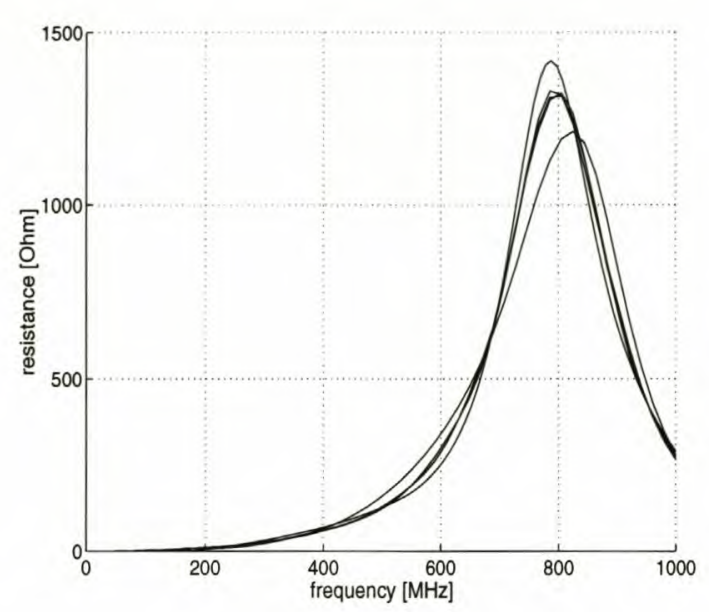

(a) real of $Z_{\text {diff }}$

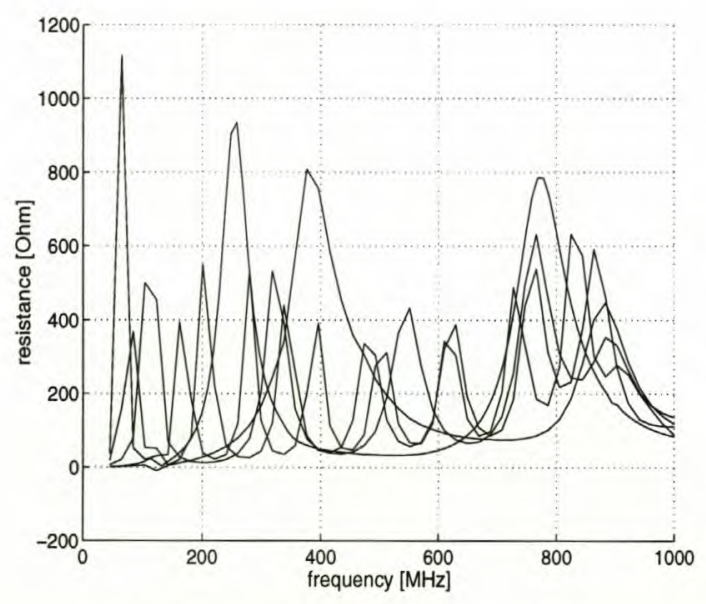

(c) real of $Z_{\text {common }}$

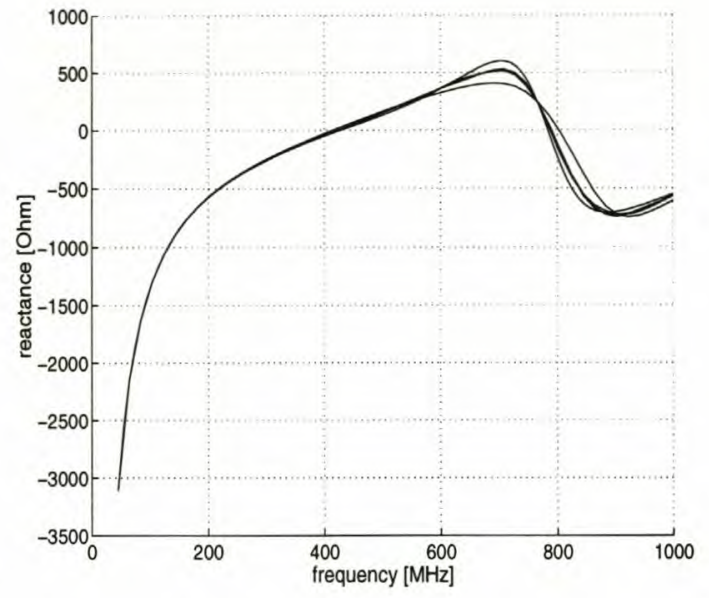

(b) $\operatorname{imag}$ of $Z_{\text {diff }}$

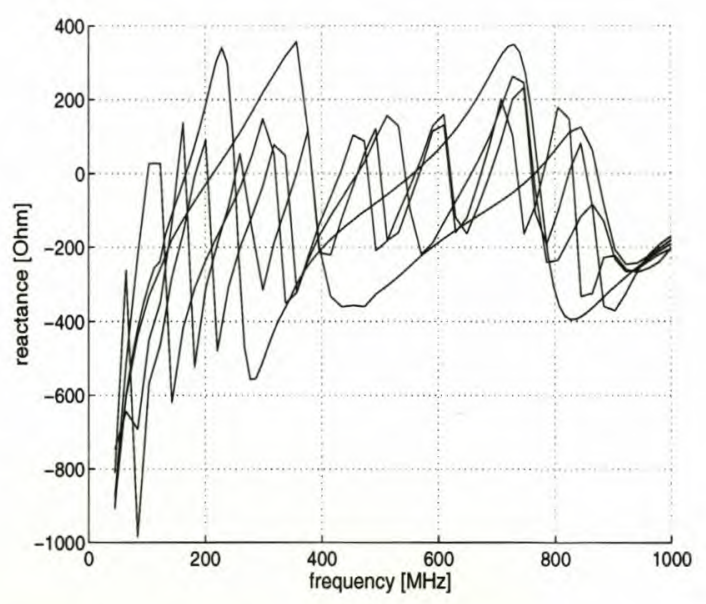

(d) imag of $Z_{\text {common }}$

Figure 4.7: The impedance of the dipole for different heights above the EMC box

\subsubsection{Height of the DUT above the EMC box}

To reduce reflections, the EMC box was designed at a 45 degree angle with respect to the antennas under test. An investigation into whether reflections from the EMC box still significantly influenced the antenna impedances was necessary. FEKO was used to simulate the effects of various distances between the dipole and EMC box. It was not deemed necessary to confirm the simulations with measurements since it was satisfactorily established in chapter 3 that FEKO is well capable of simulating such a structure. In addition, accurate values were not important since it was only necessary to determine qualitatively whether there was a significant influence. The height of the dipole was varied between $15 \mathrm{~cm}$ lower, and $1 \mathrm{~m}$ higher than the initial height. Figure 4.7 shows curves for the differential and common mode impedance for different heights of the dipole above the EMC box. 


\subsubsection{Stray inductance at the connection point with DUT}

It was often unavoidable that a finite length of the centre conductors remained after the connections were made. In this section the effect of this finite length of the centre conductors is investigated.

Usually, when antennas are measured, the effect of a finite piece of the centre conductor of the cable feeding the length of the antenna can be subtracted from the antenna impedance as follows: A very rough rule of thumb is that every $1 \mathrm{~mm}$ of length causes a series inductance of $1 \mathrm{nH}$. The corresponding reactance is then obtained by multiplying this value by the frequency in radians/sec. This reactance is then simply subtracted from the input impedance of the antenna. In the case of the measurement system proposed here, the input impedance is not directly measured and a different procedure has to be followed. This is due to the manner in which the antenna impedances are obtained - the impedances are obtained by a combination of the Y-parameters of a two-port measurement, as well as the extraction of an unwanted element (the semi-rigid cables). Instead, the unwanted reactance should be subtracted directly from the extracted Y-parameters (or Z-parameters) of the antenna. Since the frequencies at which the spiral antenna was measured were higher than those of the dipole, it was reasonable to assume that the effect of the finite length would be more significant for the spiral than for the dipole. Due to the finite thickness of the dielectric under the spiral, approximately $1 \mathrm{~mm}$ of centre conductor remained exposed at the connection. To compensate, a reactance of $j w\left(10^{-9}\right)$ was subtracted from each of the Z-parameters, Z11, Z21, Z12 and Z22 and the result was compared to the original values. Only the imaginary part of the common mode impedance showed a noteworthy variation as shown in figure 4.8. As expected, negligible differences were seen in the dipole case and are not presented here.

\subsection{Sensitivity analysis over a parameter range}

This section aims to identify where and how improvements can be made in the measurement system to achieve more accurate results. To facilitate this goal, a sensitivity analysis was performed whereby the measured S-parameters and parameters for the semi-rigid cables were varied over arbitrary values. The range of values chosen was limited to practical and realistic values.

\subsubsection{Choosing practical values for the analysis}

In choosing the S-parameters, $S_{11}, S_{21}, S_{12}, S_{22}$, two equations were selected that need to be satisfied :

$$
\begin{array}{r}
\left|S 11_{\text {all }}\right|^{2}+\left|S 21_{\text {all }}\right|^{2}=1 \\
\text { or }\left|S 11_{\text {all }}\right|^{2}+\left|S 21_{\text {all }}\right|^{2} \leq 1
\end{array}
$$

Equation 4.21 is true in all lossless, passive networks. Equation 4.22 is true in networks where losses are present. In this case, the antenna radiation causes the losses. Note that 


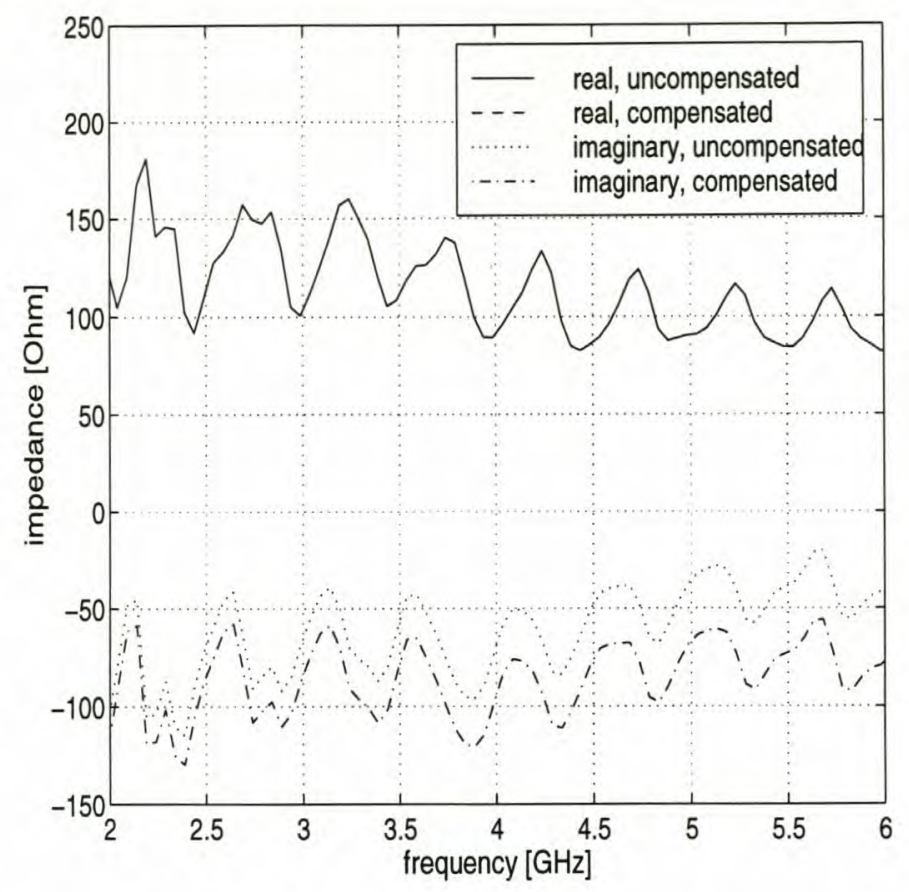

Figure 4.8: Comparison of the common mode impedance of the spiral when the stray reactance caused by the feeds are subtracted from the measurements

only the parameters S11 and S21 are mentioned. Intuitively, S12 and S22 are assumed to be the same as S21 and S11 respectively due to the nature of the antennas under test. A realistic value was necessary for the right hand side of equation 4.22 and was chosen by using the measured dipole S-parameters. Figure 4.9 shows the comparison between the magnitude of $S 11_{\text {all }}$ as measured for the dipole and $\left|S 11_{\text {all }}\right|^{2}+\left|S 21_{\text {all }}\right|^{2}$. In general the curve for $\left|S 11_{\text {all }}\right|^{2}+\left|S 21_{\text {all }}\right|^{2}$ is some 10 percent lower than the curve for $\left|S 11_{\text {all }}\right|$. From this figure the expression on the right hand side of equation 4.22 was chosen to be $0.9\left|S 11_{\text {all }}\right|$. When performing the analysis, the following variables and their variations were considered :

1. The measured S-parameters were varied between well matched and badly matched values. Specifically $S 11_{\text {all }}$ and $S 22_{\text {all }}$ were selected to be $-1 \mathrm{~dB},-6 \mathrm{~dB},-20 \mathrm{~dB}$, and $S 21_{\text {all }}$ and $S 12_{\text {all }}$ were calculated using (4.22).

2. The semi-rigid parameters, $\alpha$ and $\beta$, were varied between a tenth and twice their measured values as determined by the measurements and results in chapter 3 .

\subsubsection{The sensitivities for the S-parameters and the semi-rigid cables}

Figures 4.10 and 4.11 show the sensitivities for the magnitude and angle of $Z_{\text {diff }}$ and $Z_{\text {common }}$ for the chosen S-paramaters, $S_{\text {all }}$. Each subfigure shows the sensitivity for different values of the semi-rigid parameters. 


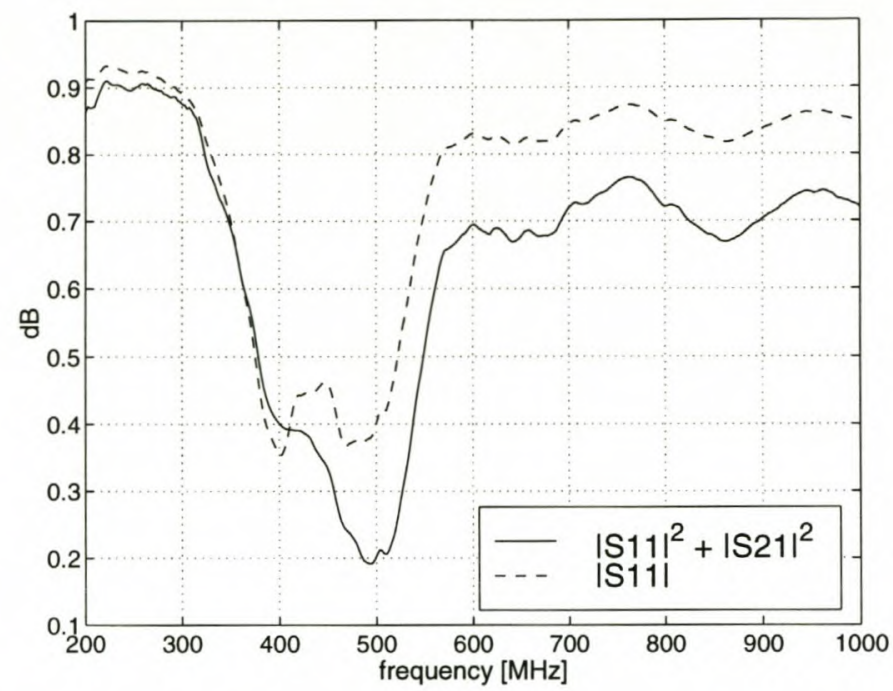

Figure 4.9: Comparison between $\left|S 11_{\text {all }}\right|$ and $\left|S 11_{\text {all }}\right|^{2}+\left|S 21_{\text {all }}\right|^{2}$ as measured for the dipole

Results were presented for the S-parameters chosen using equation 4.22. Although not shown, the sensitivities for the S-parameters chosen using equation 4.21 were lower than those shown here. This result is however of no practical importance since it is not physically meaningful to alter the radiation properties, and hence the S-parameters, of the antenna in order to achieve lower sensitivity. The analysis using 4.21 was performed merely for interest. 


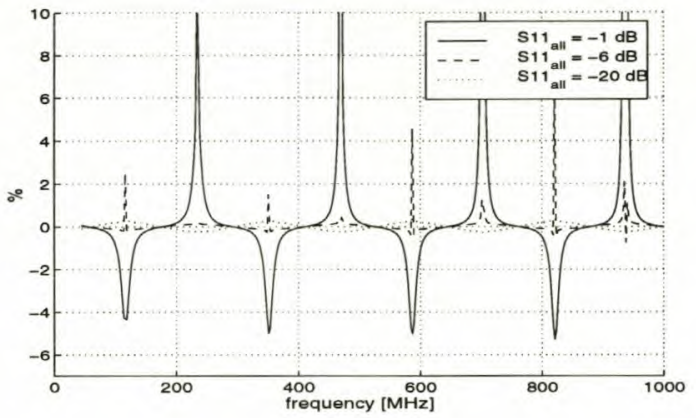

(a) $\left|Z_{\text {diff }}\right|$ for $\alpha, \beta=1 \mathrm{x}$

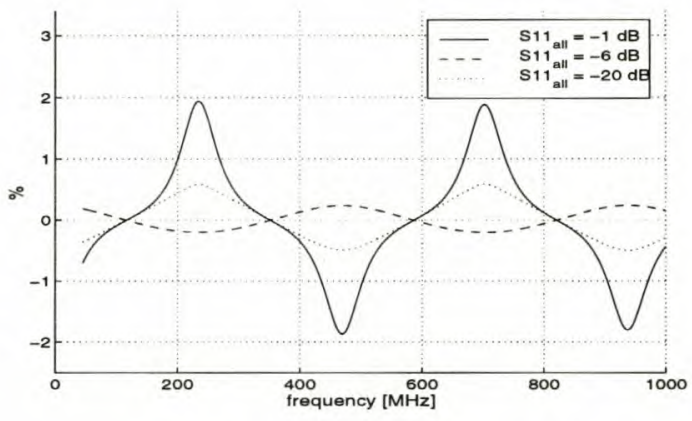

(c) $\left|Z_{\text {diff }}\right|$ for $\alpha, \beta=0.5 \mathrm{x}$

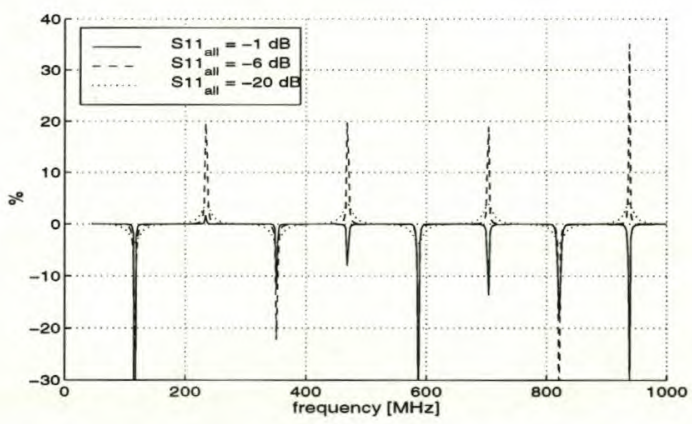

(e) $\left|Z_{\text {common }}\right|$ for $\alpha, \beta=1 \mathrm{x}$

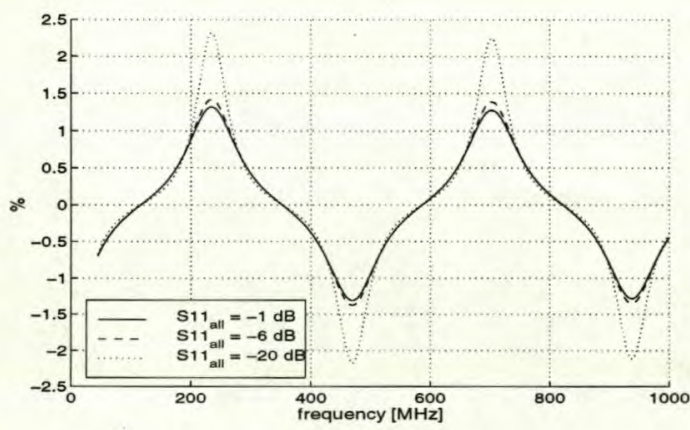

(g) $\left|Z_{\text {common }}\right|$ for $\alpha, \beta=0.5 \mathrm{x}$

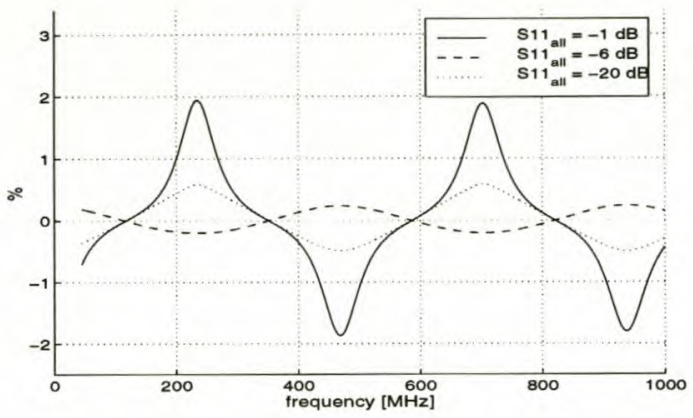

(b) $\left|Z_{\text {diff }}\right|$ for $\alpha, \beta=2 \mathrm{x}$

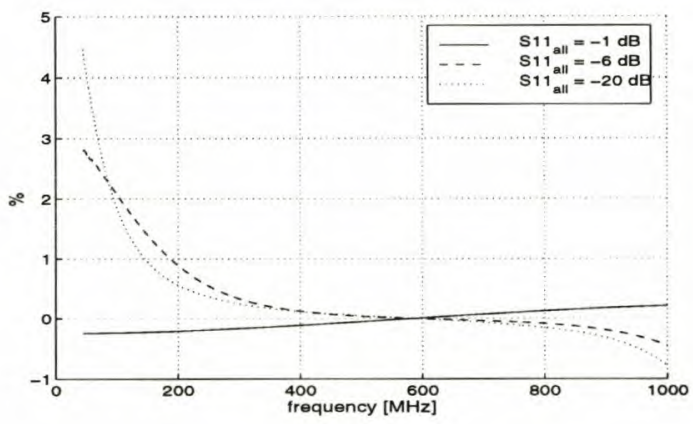

(d) $\left|Z_{\text {diff }}\right|$ for $\alpha, \beta=0.1 \mathrm{x}$

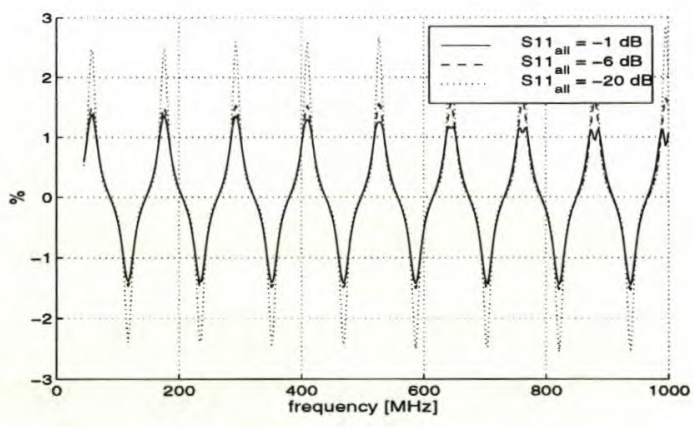

(f) $\left|Z_{\text {common }}\right|$ for $\alpha, \beta=2 \mathrm{x}$

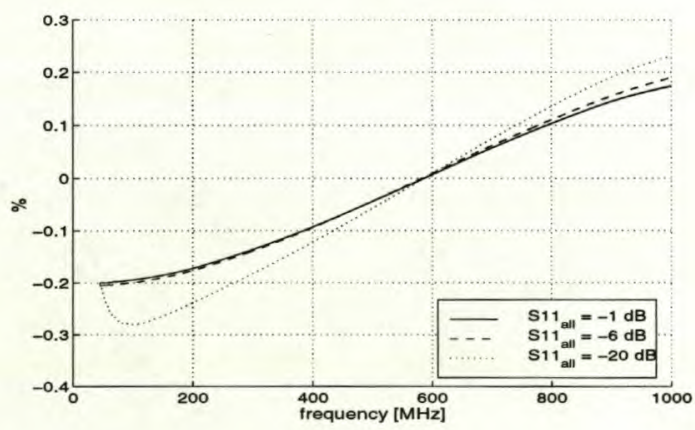

(h) $\left|Z_{\text {common }}\right|$ for $\alpha, \beta=0.1 \mathrm{x}$

Figure 4.10: Sensitivity of $\left|Z_{\text {diff }}\right|$ and $\left|Z_{\text {common }}\right|$ for generally chosen S-parameters using the measurement jig 
CHAPTER 4. SENSITIVITY ANALYSIS

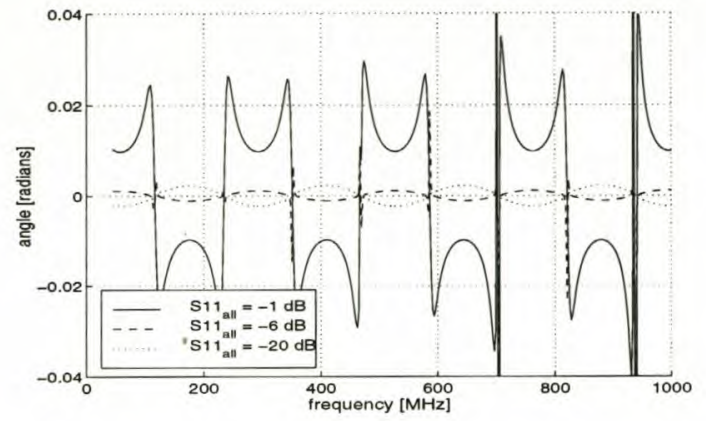

(a) angle $Z_{\text {diff }}$ for $\alpha, \beta=1 \mathrm{x}$

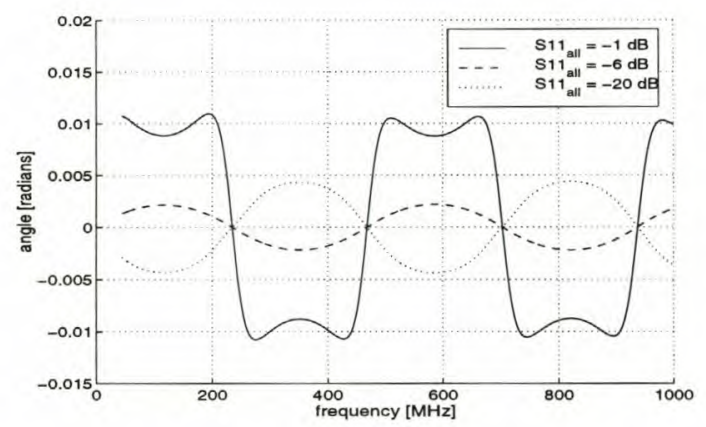

(c) angle $Z_{\text {diff }}$ for $\alpha, \beta=0.5 \mathrm{x}$

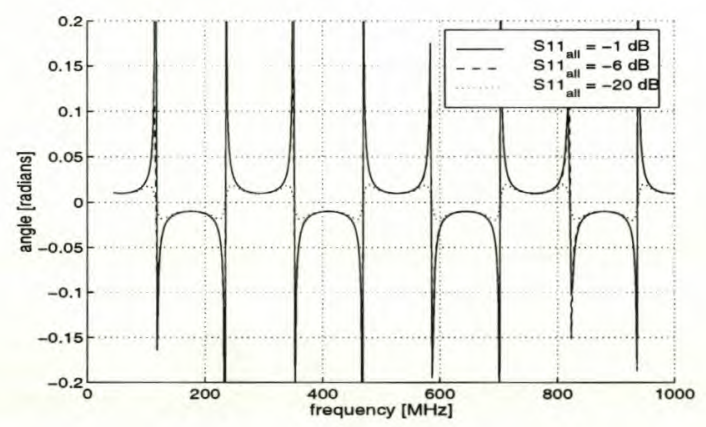

(e) angle $Z_{\text {common }}$ for $\alpha, \beta=1 \mathrm{x}$

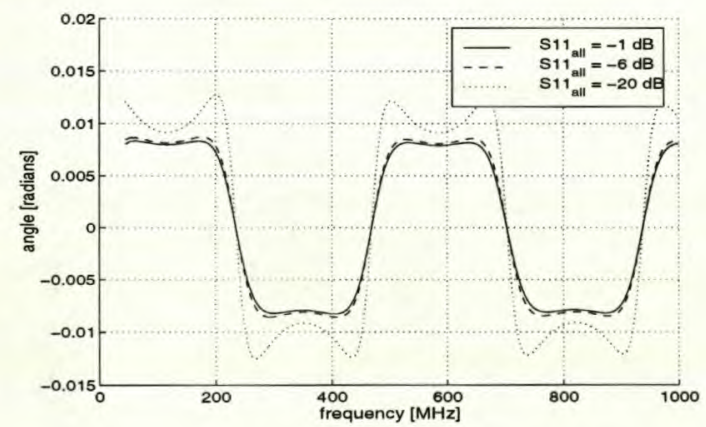

(g) angle $Z_{\text {common }}$ for $\alpha, \beta=0.5 \mathrm{x}$

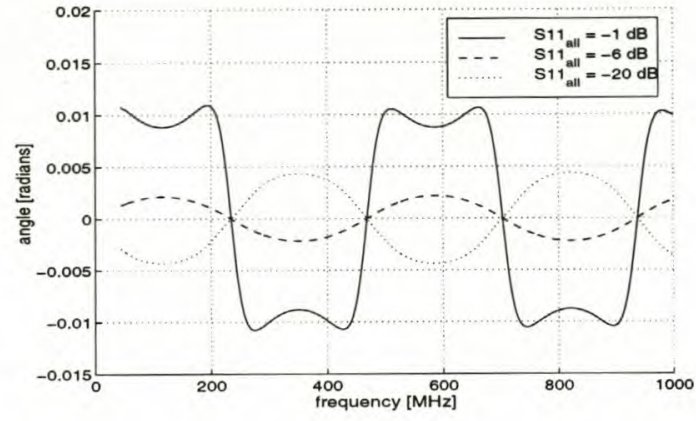

(b) angle $Z_{\text {diff }}$ for $\alpha, \beta=2 \mathrm{x}$

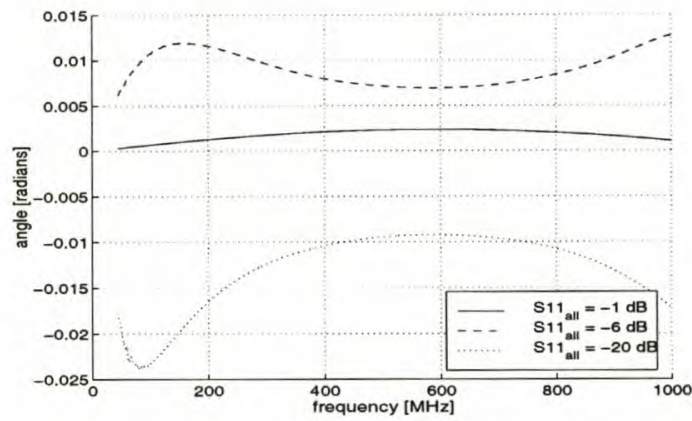

(d) angle $Z_{\text {diff }}$ for $\alpha, \beta=0.1 \mathrm{x}$

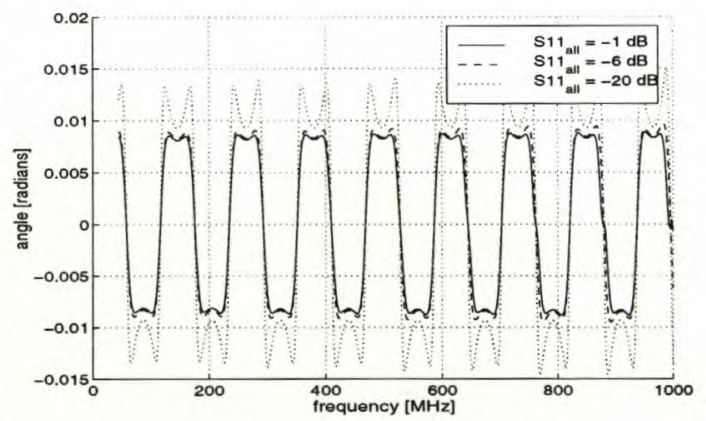

(f) angle $Z_{\text {common }}$ for $\alpha, \beta=2 \mathrm{x}$

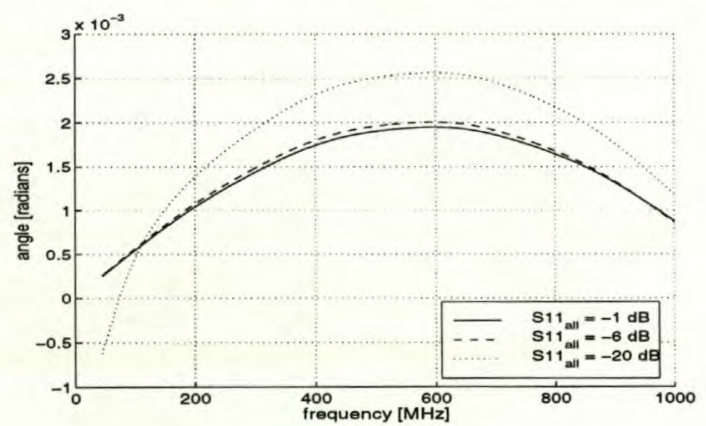

(h) angle $Z_{\text {common }}$ for $\alpha, \beta=0.1 \mathrm{x}$

Figure 4.11: Sensitivity of the angle of $Z_{\text {diff }}$ and $Z_{\text {common }}$ for generally chosen Sparameters 


\section{Chapter 5}

\section{Interpretation of sensitivities and improvements}

In this chapter the sensitivity analysis performed in chapter 4 is interpreted to determine whether improvements in the measurement system can be made to achieve greater accuracy.

\subsection{Interpretation of the sensitivity analysis of the mea- sured data}

The first subject of study was the sensitivities of the antennas, semi-rigid cables and general setup in chapter 3 .

\subsubsection{The sensitivity of the dipole antenna impedances in terms of the measured S-parameters}

When figure 4.3 and the figures for the differential (fig.3.12) and common mode impedance (fig.3.13) of the dipole are compared, it can clearly be seen that where the discrepancies between measurement and simulation are the greatest, the sensitivities are the greatest as well. In general, it is seen that high sensitivities are correlated with high antenna impedances. Although the angle sensitivities are not expressed in their normalized form (as a percentage), one can assume that due to their very low values, the influence of the angle changes on the antenna impedances are negligible.

\subsubsection{The sensitivity of the spiral antenna impedances in terms of the measured S-parameters}

As with the dipole, there was a direct correlation between the impedance value and the sensitivity. (The periodic behaviour of the curves will be dealt with later). Again it 
can be seen that high sensitivities are associated with high antenna impedances. This is advantageous for antenna measurements since it is usually only necessary to make very accurate measurements in the operating band of the antenna where the antenna impedance is sufficiently low.

\subsubsection{The sensitivity of the impedances to the semi-rigid cables}

From, for example, figure 4.6((a)) it is seen that the antenna impedances are not sensitive to the losses in the semi-rigid cables. The sensitivity to the length of the cable is very high (see, for example, figure (e)). Looking at the magnitude of the impedances, there is again a correlation with the sensitivity curves as was the case with the measured S-parameters shown in the previous section. The sensitivity curves for the spiral antenna are again somewhat periodic and will be the subject of discussion further on.

\subsubsection{Height of the DUT above the EMC box}

It is seen from fig. 4.7 that the differential impedance is not very sensitive to reflections from the EMC box. Rather, it is the common mode impedance that is most strongly influenced by the separation between the dipole and the EMC box. This was to be expected due to the flow of common mode current on the outside of the outer conductors of the semi-rigid cables.

\subsection{Interpretation of the sensitivity analysis over a pa- rameter range}

In this section the results of the general sensitivity analysis are interpreted.

\subsubsection{Sensitivities for the S-parameters and the semi-rigid cables}

From figures 4.10 and 4.11 it is seen that the sensitivity of the differential impedance is lower when the DUT (the antenna plus the semi-rigid cables combination) is well matched $(\mathrm{S} 11=-20 \mathrm{~dB})$. This is not the case for the common mode impedance, where the opposite is true, although not as pronounced as in the case of the differential impedance. When these graphs are compared for the different parameters of the semi-rigid cables, it is seen that dropping $\alpha$ and $\beta$, lowers the sensitivity. In most cases, dropping $\alpha$ and $\beta$ to a tenth of their original values (figures (d) and (h)) drops the sensitivity significantly.

\subsubsection{The periodicity of the sensitivities}

It was seen earlier that the impedance of the spiral antenna demonstrated periodic behaviour. This was also seen in the previous section where the generalized sensitivities 
were shown. From Figures 4.10 and 4.11 it is seen that the number of peaks in the curves are correlated with the multiplication factor of $\alpha$ and $\beta$. That is, the number of peaks increase when $\alpha$ and $\beta$ are increased, and they decrease vice versa. Since the input S-parameters, $S_{\text {all }}$, were chosen to be constant for all frequencies, it was reasonable to investigate whether there was a resonance effect present due to the fixed physical length of the semi-rigid cables.

The physical lengths of the semi-rigid cables were $44 \mathrm{~cm}$. Due to the fact that the propagation velocity in the coaxial cable is a third lower than in free space, its effective length was assumed to be $66 \mathrm{~cm}$. The frequency corresponding to this wavelength in the semi-rigid cables is $454 \mathrm{MHz}$. The input impedance of the spiral, fig. 3.8, indeed showed maxima and minima at increments of $234 \mathrm{MHz}$ respectively, or every $\frac{\lambda}{2}$ of the semi-rigid cables. This was also evident in the case of the generalized sensitivity analysis.

\subsection{Conclusions}

The sensitivity analysis performed in this chapter has proved to be advantageous in that it has shown the following:

- Use of short semi-rigid cables can reduce the overall sensitivity, specially to the parameters of the semi-rigid cables as well as minimize or eliminate periodicities in the extracted impedances due to resonances in the semi-rigid cables

- Although the use of an EMC box provides better control over the common mode current flow, it reflects the radiated waves back to the antenna causing discrepancies in the antenna impedance, specially in the common mode impedance

- When characterising the semi-rigid cables, it is very important to minimize errors in measurement of the electrical length of the semi-rigid cables

- It is not necessary to use expensive low loss semi-rigid cables due to the very low sensitivity of the results for the loss in the semi-rigid cables

- Very accurate measurements of the S-parameters of the full measurement are not necessary unless high accuracy is also required outside the operating band of the antenna (where the input impedances are high)

- The stray inductance often present at the antenna connection points can be ignored for frequencies of at least up to $7 \mathrm{GHz}$.

With the focus on the differential input impedance and based on the above findings, changes were made in the measurement system in order to obtain more accurate measurements: The EMC box was no longer used and the semi-rigid cables were made as short as possible.

In the following chapter, final results are presented where the above changes were implemented. 


\section{Chapter 6}

\section{Final results}

In this chapter final results are presented for the spiral and dipole antennas where the improved measurement jig was used. Measurements of a bow tie antenna are also presented.

\subsection{Simulation in FEKO}

It was deemed necessary to simulate the changed system used in the measurements. Specifically, the absence of the EMC box necessitated the simulation. FEKO was used for both the spiral and dipole antenna due to FEKO's ability to load segments with arbitrary impedances.

Since an infinitely long cable can not be simulated in FEKO, a truncated wire was used to model the coaxial cables used in the measurements. To prevent standing waves on the wire in FEKO, it was required to stop or, at least, suppress the flow of the common mode currents on the wire. A realistic manner of doing this was to stop the current only nearer to the end of the wire, furthest from the antenna as to accurately model the actual measurement layout. For this purpose the Wu-King resistive loading profile [8],[9] was used to stop the common mode current flow on the semi-rigid cables.

\subsection{The Wu-King profile}

The Wu-King profile is a continuous impedance profile which results in a pure outwardtraveling wave of current (no reflections) on a finite length of wire. The impedance profile is a function of the axial coordinate, $\mathrm{z}$, and is given by

$$
Z^{i}(z)=\frac{60 \psi}{h-|z|}
$$

where $h$ is the length of one antenna arm, $\psi$ is the complex expansion parameter and $z$ the axial coordinate.

This profile is generally used in impedance loaded antennas and results in a wide-band antenna, typically of low efficiency. 


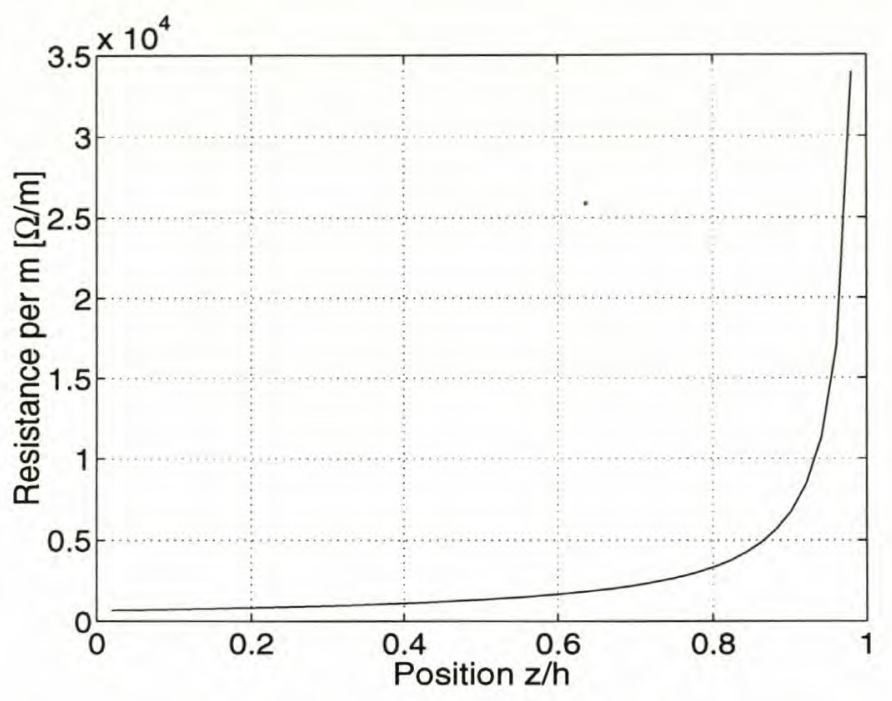

Figure 6.1: Resistance required along the semi-rigid cable to stop the current in FEKO

\subsection{The dipole antenna}

The dipole antenna was simulated using a very long and thick wire representing the semirigid and coaxial cables. The segments of the wire nearer to the antenna were left unloaded while the segments near the end of the wire were loaded using the Wu-King profile. The wire was $7 \mathrm{~mm}$ thick and $1 \mathrm{~m}$ long. Figure 6.1 shows the resistances that the Wu-King profile (6.1) produced. The figure shows the resistances for the normalized length of the loaded section of the wire.

Initially, standard coaxial cables of $1 \mathrm{~m}$ length were used as well as for the measurements shown in chapter 3 (besides the short semi-rigid cables already mentioned). It was found during measurement that when the position of the coaxial cables was changed, the measurements also changed. For example, two sets of measurements were taken where only the orientation of the cables was changed. The resulting differential and common mode impedances of the dipole are shown in figure 6.2. It is seen that the differences, even in the differential mode impedance, are highly unsatisfactory. The discrepancies were very likely due to the common mode current flow. It is also possible that the transfer impedance of the coaxial cables were not sufficiently high.

A different pair of coaxial cables, approximately $3 \mathrm{~m}$ long, was then used. Unlike the first set of coaxial cables, these cables were equipped with armoured shielding. The measurements were repeated and fig. 6.3 shows the new comparisons. It is seen that the differential impedance remained satisfactorily stable, but as expected the common mode impedance is a function of how the cables are routed.

To confirm the accuracy of the measurements, the dipole was also measured in the conventional manner where a ground plane and the method of images is used. Fig. 6.4 shows the simulated vs. measured results. It is seen that the two measurements compare very well in form and value. The FEKO simulation shows only a slight shift in frequency a common occurrence when numerical tools are used to model antennas. The common mode impedance of the dipole did not compare satisfactorily to that of the simulation 


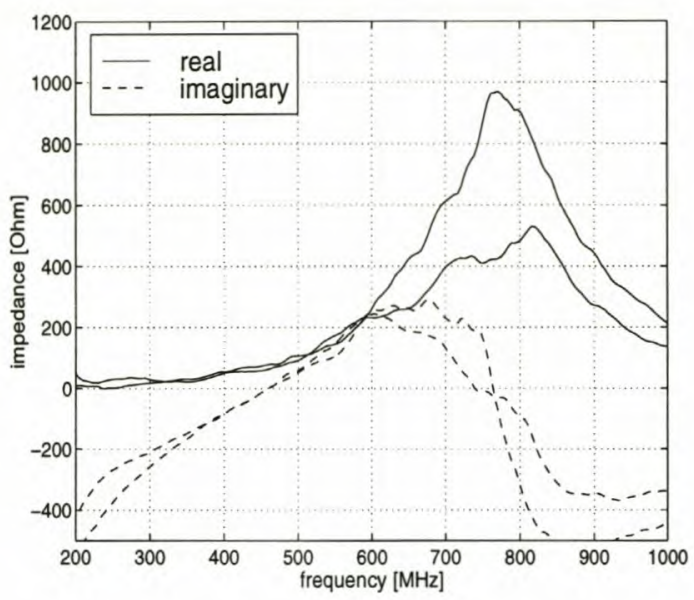

(a) Zdifferential

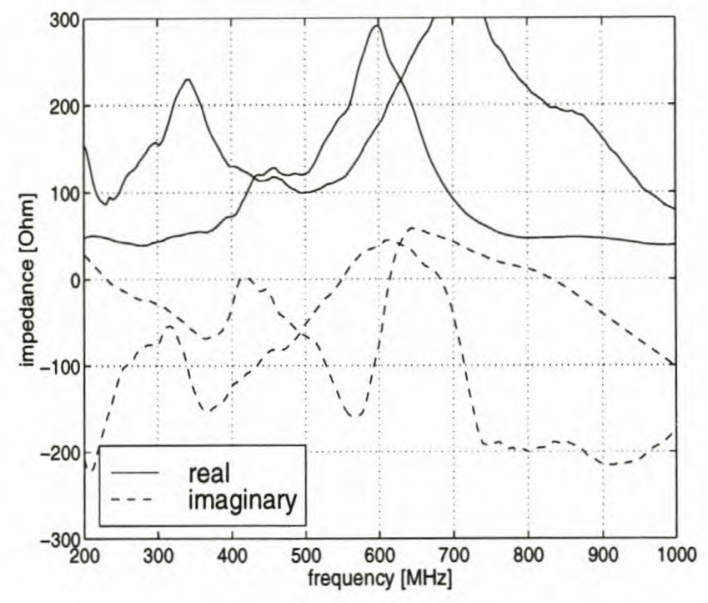

(b) Zcommon

Figure 6.2: Comparison of the differential and common mode impedance of the dipole as measured with a short, standard pair of coaxial cables where only the orientations of the cables were changed.

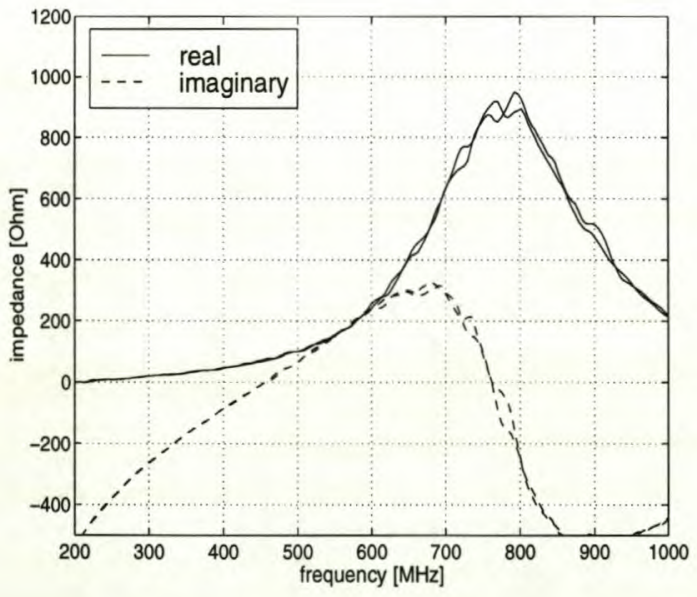

(a) Zdifferential

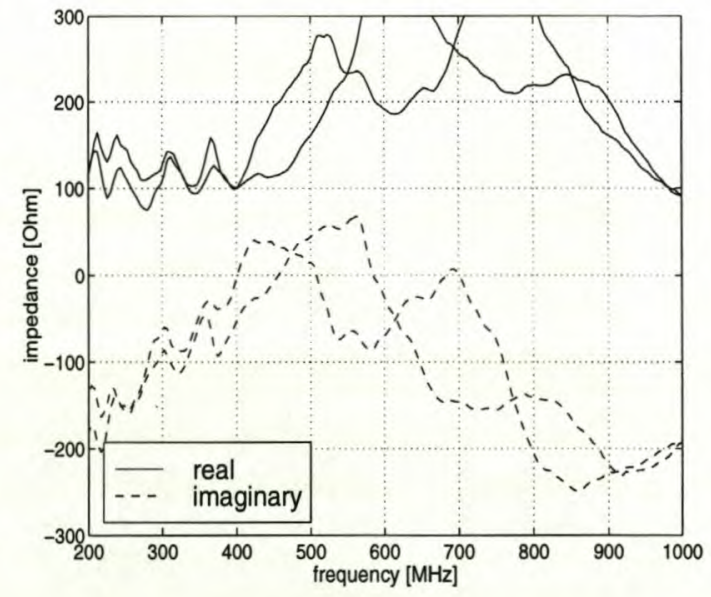

(b) Zcommon

Figure 6.3: Comparison of the differential and common mode impedance of the dipole as measured with a longer pair of coaxial cables where only the orientations of the cables were changed. 


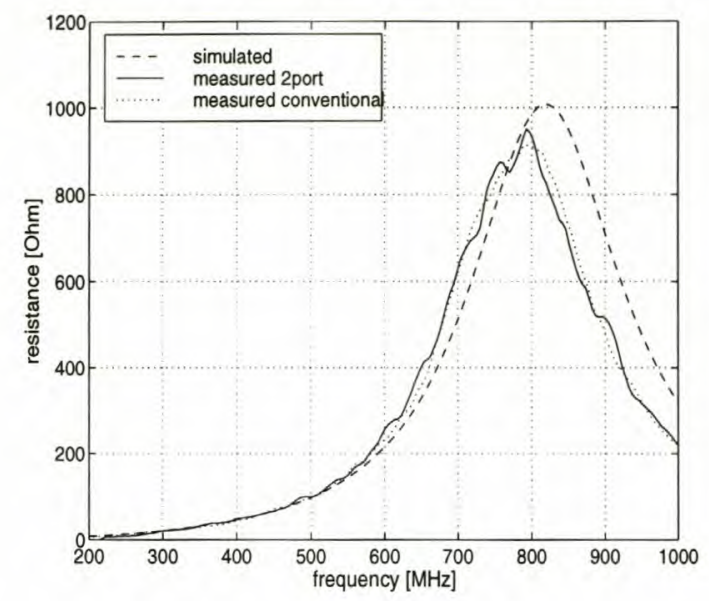

(a) real part

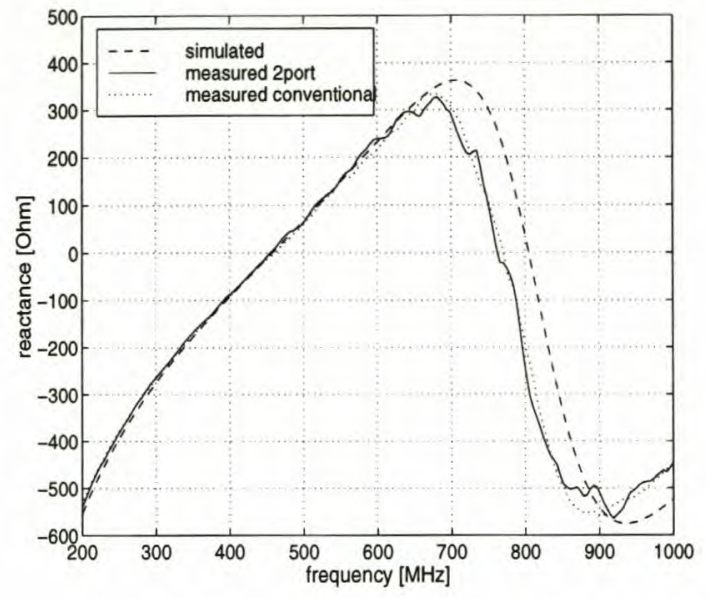

(b) imaginary part

Figure 6.4: Comparison of the differential input impedance of the dipole as measured using two methods and simulated in FEKO.

and is not shown.

The results for the dipole highlighted the influence and difficulties arising from the common mode current flow. By removing the EMC box from the measurements, increased difficulty in accurately modeling the whole measurement layout was experienced- implementing the Wu-King Profile did not prove successful in that the common mode current flow could not be accurately modelled.

\subsection{The spiral antenna}

The spiral was remeasured using the longer pair of coaxial cables. Figure 6.5 shows the measured result as well as the simulated result of chapter 3 where only 1 port was used in the simulation.

Compared to the previous measurement (fig. 3.8), improvement is evident by observing the following :

- The periodic behaviour of the curves are less pronounced.

- The real part of the input impedance is more comparable in form to the curve of the simulation

- The imaginary part also shows better agreement in value, specially at the top of the band.

As was seen in chapter 3 in fig. 3.8 , the measurement as well as the 2 port simulation showed a rising imaginary part. This was thought to be due to the manner in which the spiral was fed - in the measurement and the 2 port simulation the connections were not 


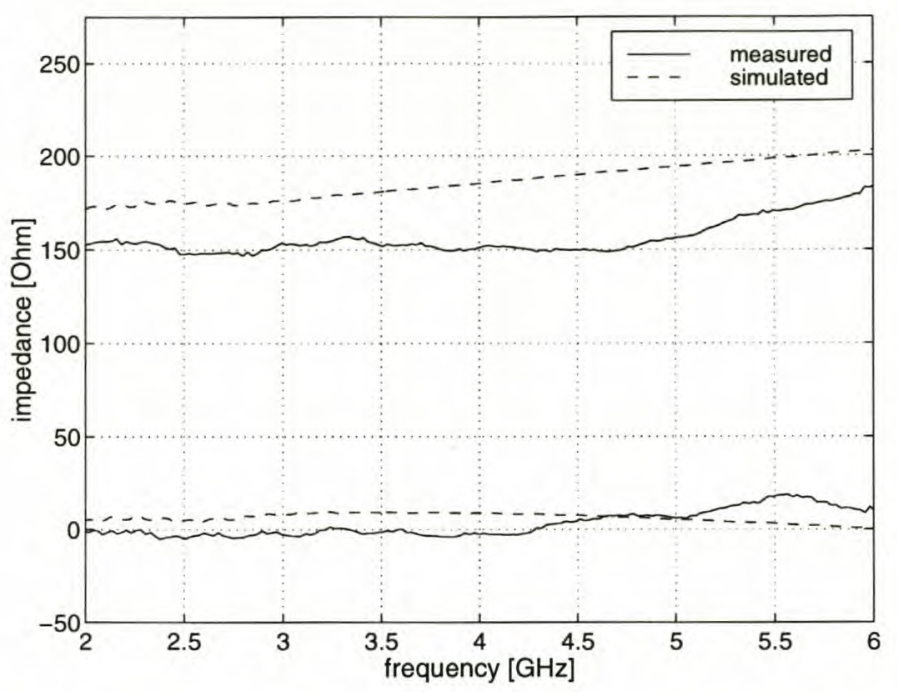

Figure 6.5: Measured vs. simulated result using the short semi-rigid cables and the coaxial cables with higher transfer impedance.

made in the middle between the spiral arms, whereas in the 1 port simulation the single feed point was centered. Nevertheless, the new measurement showed improvement in that the increase in the imaginary part with frequency was less pronounced.

\subsection{A bow tie antenna}

A bow tie antenna was manufactured and measured as a final example. This was deemed useful to further show the reliability of the proposed method since the bow tie can also be measured in the conventional manner where a ground plane and the method of images is used.

The geometry of the bow tie antenna is shown in fig. 6.6. The flare angle was 90 degrees and the hypotenuses were of equal length.

The bow tie antenna was measured using the two methods. The first was the conventional method where the method of images is used - only one "wing" of the bow tie is used with a ground plane to provide the mirror image of the "wing". The measured input impedance is then multiplied by 2 to find the differential input impedance. The 2nd method was the proposed two port measurement. Fig. 6.7 shows the two methods. Figure 6.8 shows the measured curves for the differential input impedance. The first curve is the measured input impedance using the 2 port jig. The other curve is the measured input impedance using the conventional method. The two measured curves are in very good agreement proving again the reliability and accuracy of the proposed two port method. No deterioration in accuracy with rising frequency is visible. 


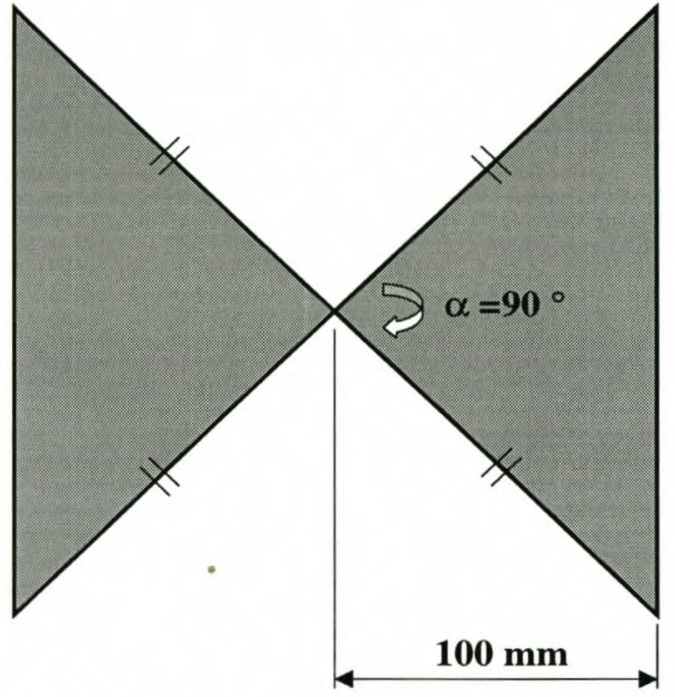

Figure 6.6: The bow tie antenna

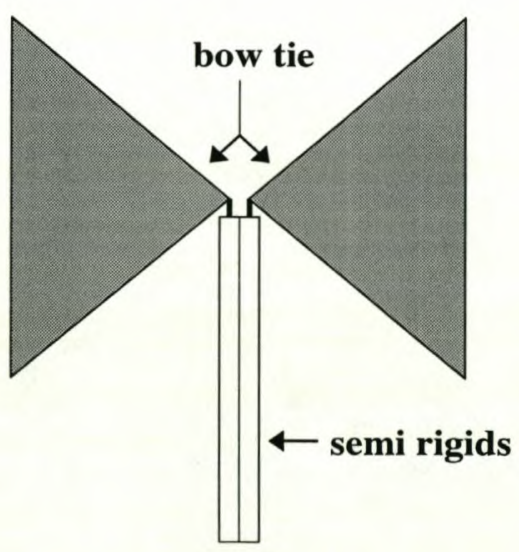

(a) 2 port jig

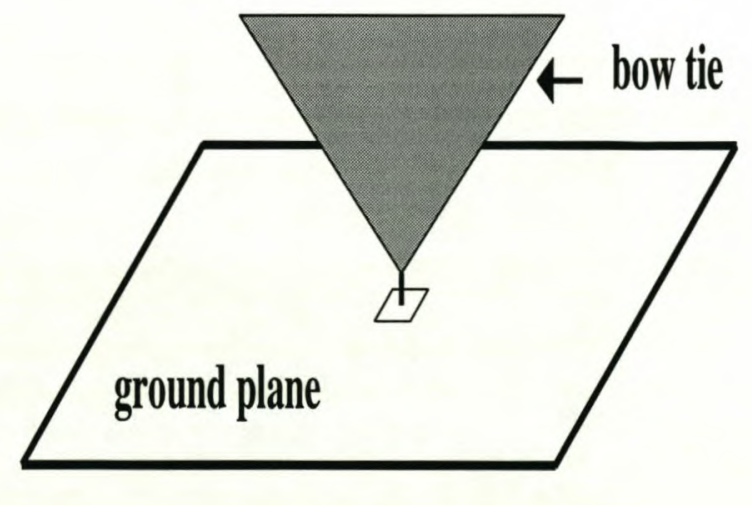

(b) conventional

Figure 6.7: The two methods used to measure the bow tie antenna 


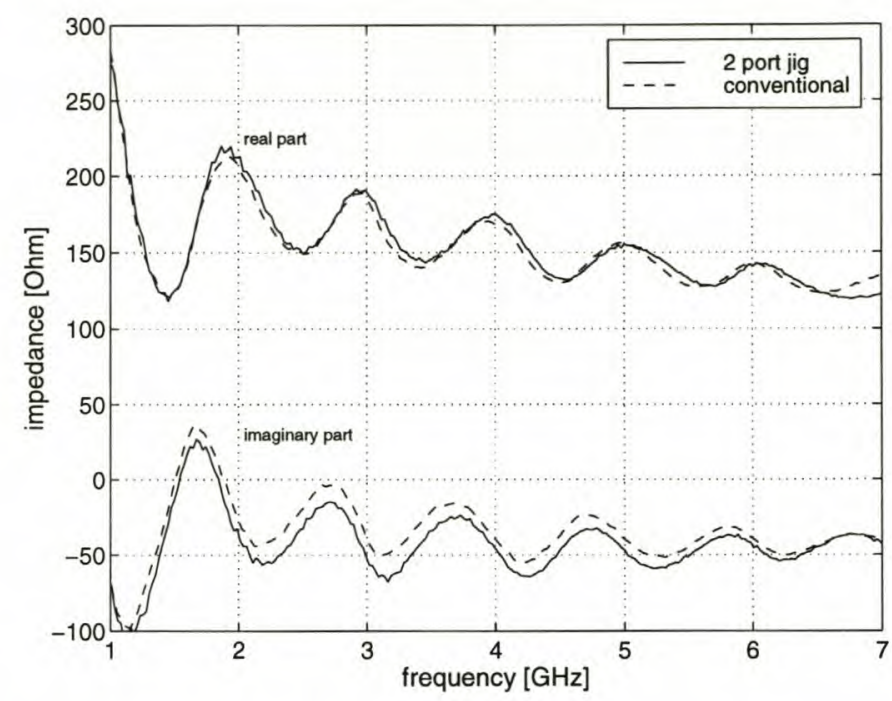

Figure 6.8: Differential input impedance of the bow tie antenna using different measuring techniques

\subsection{Sensitivities using the modified jig}

In this section the sensitivities using the modified jig (the short semi-rigid cables and the long coaxial cables) will be presented.

\subsubsection{Sensitivities for the measured S-parameters and semi-rigid cables}

Fig. 6.9 shows the sensitivities for the measured S-paramaters. It is seen that although the sensitivities are unchanged in general, they are satisfactorily low. Fig. 6.10 shows the sensitivities for $\alpha$ and $\beta$ of the new semi-rigid cables. As seen, the sensitivities are lower by 10 to 50 times in general compared to the original jig used for measurements in chapter 3 . They are also less periodic with frequency.

\subsubsection{Stray inductance at the connection point with the DUT}

It was re-investigated whether the influence of the stray inductance caused by the exposed centre conductors remained negligible as in the case of the old measurement jig.

The spiral antenna's measurements were repeated, and the reactance caused by the centre conductors was subtracted as explained in chapter 4.

It was found that the effect was indeed negligible. Fig. 6.11 shows the effect for the common mode impedance of the spiral. The two curves for the differential impedance were indistinguishable from each other to such an extent that they were deemed unnecessary to display here. 


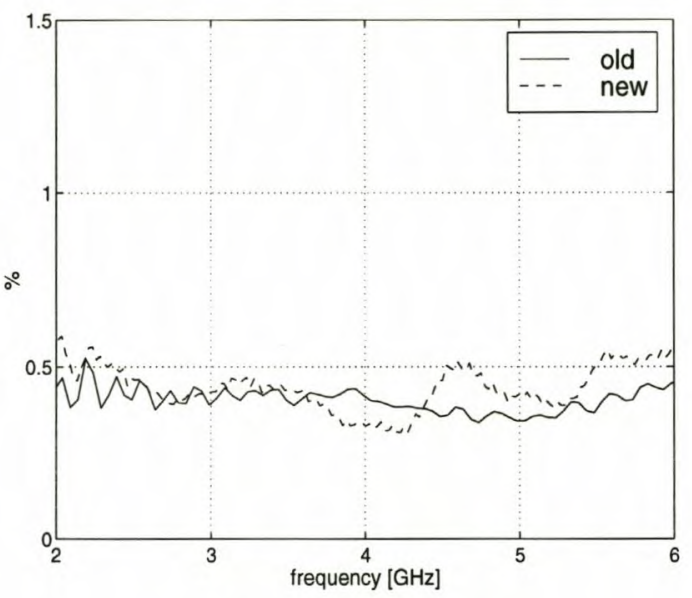

(a) $\left|Z_{\text {diff }}\right|$

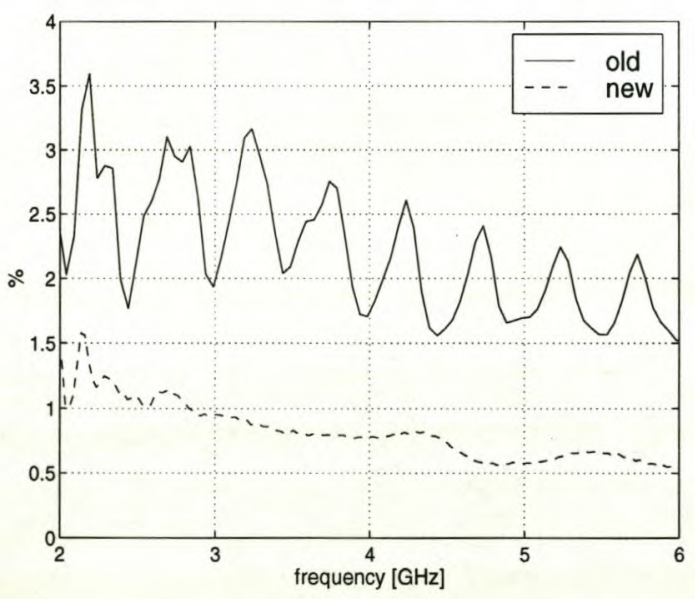

(c) $\left|Z_{\text {common }}\right|$

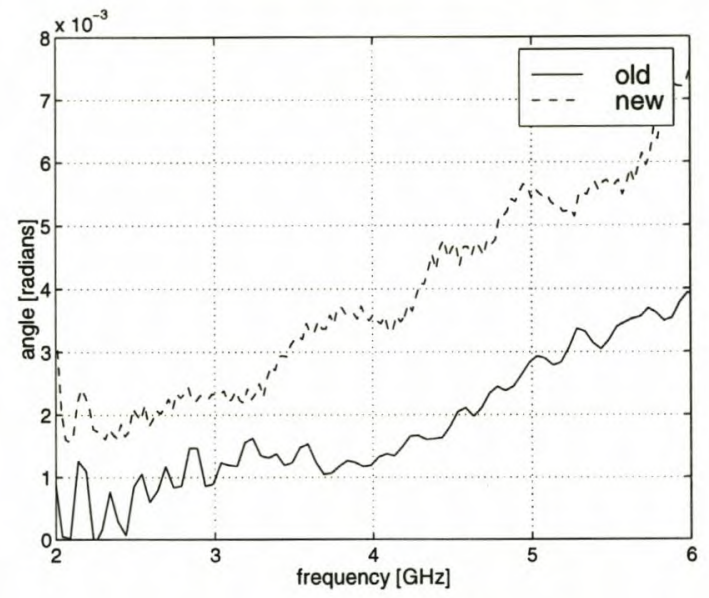

(b) angle $Z_{\text {diff }}$

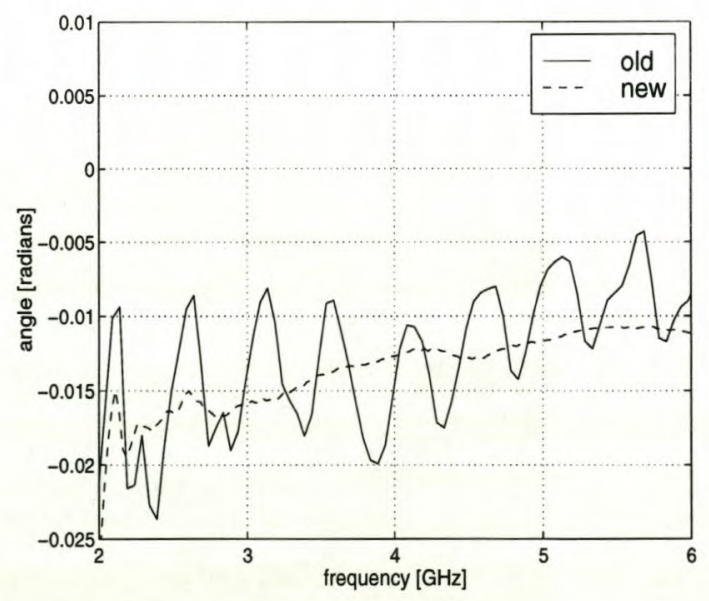

(d) angle $Z_{\text {common }}$

Figure 6.9: Sensitivity of $\left|Z_{\text {diff }}\right|$ and $\left|Z_{\text {common }}\right|$ of the spiral for the measured S-parameters using the modified jig 


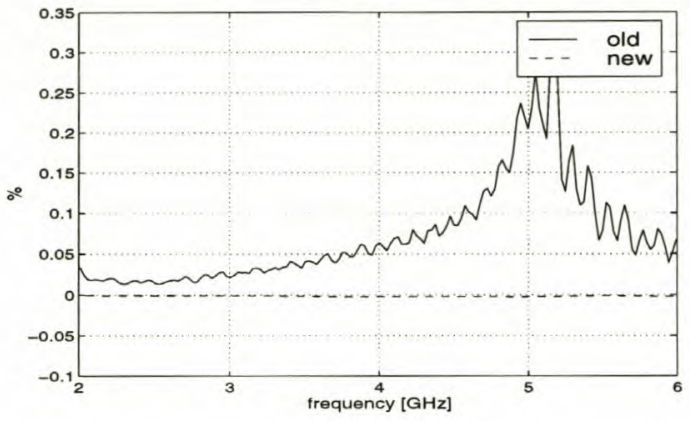

(a) $\left|Z_{\text {diff }}\right|$ for $\alpha$

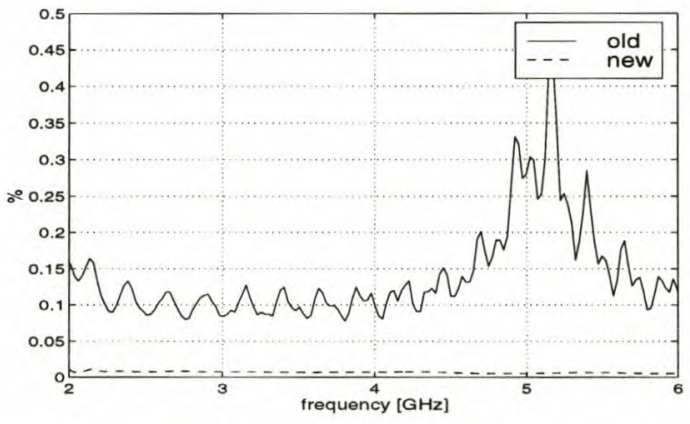

(c) $\left|Z_{\text {common }}\right|$ for $\alpha$

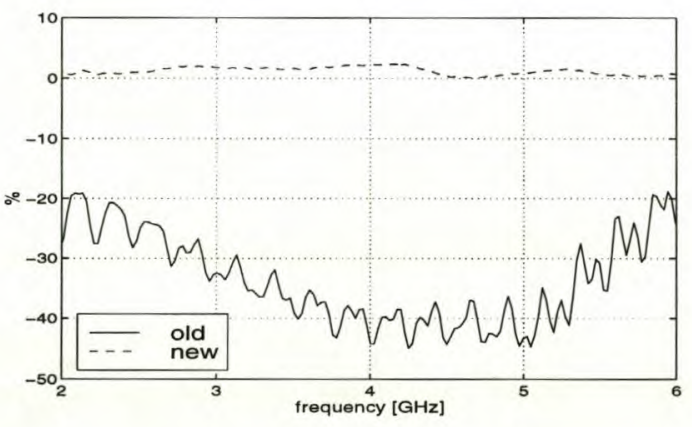

(e) $\left|Z_{\text {diff }}\right|$ for $\beta$

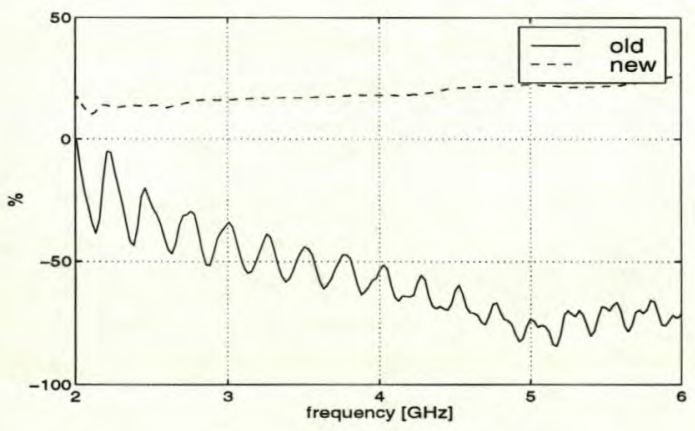

(g) $\left|Z_{\text {common }}\right|$ for $\beta$

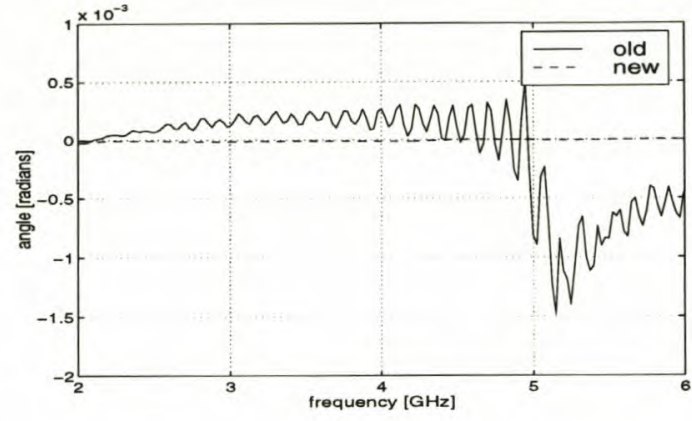

(b) angle $Z_{\text {diff }}$ for $\alpha$

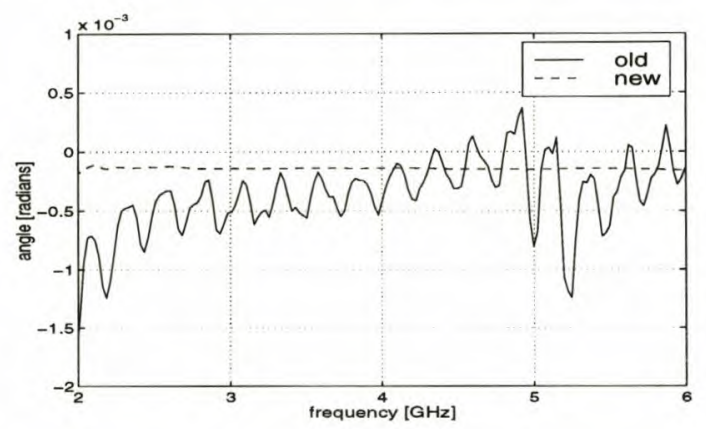

(d) angle $Z_{\text {common }}$ for $\alpha$

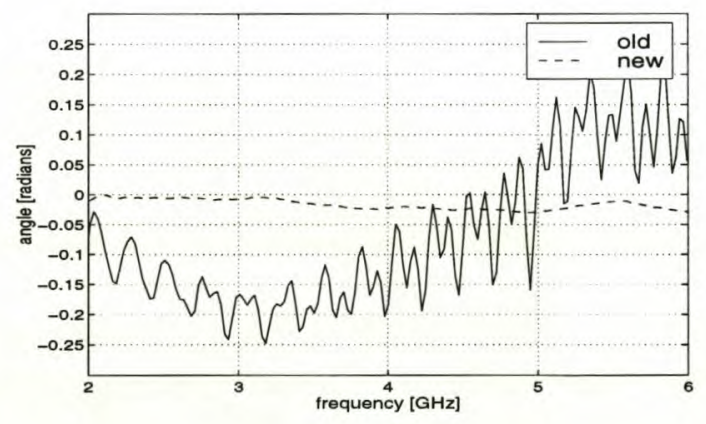

(f) angle $Z_{\text {diff }}$ for $\beta$

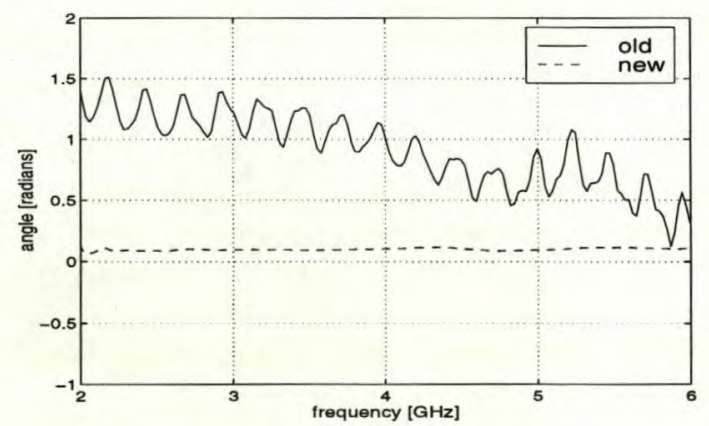

(h) angle $Z_{\text {common }}$ for $\beta$

Figure 6.10: Sensitivity of $Z_{\text {diff }}$ and $Z_{\text {common }}$ of the spiral for $\alpha$ and $\beta$ of the new semi-rigid cables 


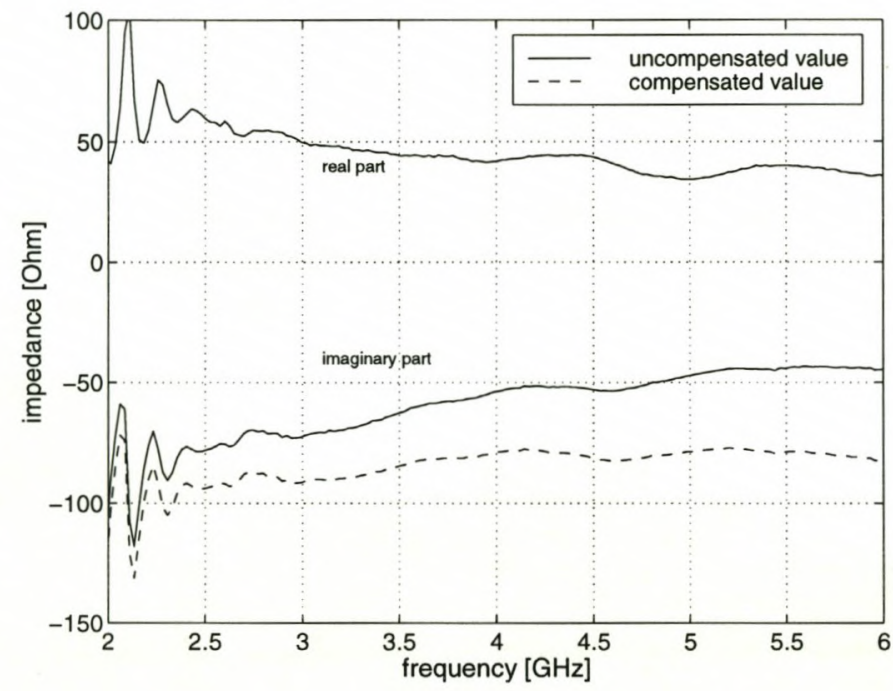

Figure 6.11: Measured spiral common mode input impedance compared to that from which the stray inductance at the feed was subtracted 


\section{Chapter 7}

\section{Conclusions}

This thesis was motivated by the need for an easier and quicker method to determine whether balanced loads such as antennas have been correctly designed and manufactured to operate in the required frequency band.

It was shown that the proposed method offered several advantages over earlier methods :

- No balun is required to measure the impedance of the antenna or balanced load. Using a balun causes uncertainty in that when a balanced load is measured, any discrepancies between the impedance that was required and that which was measured could be due to either the balun or the antenna performing incorrectly. By using this method, the uncertainty surrounding a balun can be eliminated.

- The method is not sensitive to the measurement equipment used. Standard calibration kits and semi-rigid cables can be used. A certain percentage error made in the measurements or the characterization of the semi-rigid cables will cause an error of similar magnitude in the results in most cases. In general, though, the resulting error will be reduced.

- No EMC box to shield common mode current flow is necessary if the common mode impedance is not required. In chapter 5 , it was shown that a long pair of cables was necessary to make accurate measurements. It is possible that currents induced on the outside of the coaxial cables were sufficiently attenuated at the point where the cables connected to the network analyzer, thereby minimizing any unwanted signals entering the network analyzer and causing discrepancies in the results. Although not confirmed, it is also possible that the longer pair of cables had a higher transfer impedance as it had an armoured shield to improve its ruggedness.

- The method is simple in that the semi-rigid cables that are used need only be characterized once. Thereafter, a simple computer program is stored for future use. Measured S-parameters can simply be substituted into the program to yield the antenna impedance.

- Although only shown here to be accurate up to $7 \mathrm{GHz}$, it is assumed that the method can be applied with similar accuracy at higher frequencies. 
- Although it was initially deemed necessary to use time-domain gating when measuring the semi-rigid cables to achieve higher accuracy, it was found that the results were not sensitive to errors in $\alpha$ of the semi-rigid cables. In addition, the value of $\beta$ remained the same whether or not time-domain gating was used.

The model for the antenna impedances assumed in the beginning was proven through measurement and simulation to be sufficient for obtaining the antenna differential input impedance without the use of a balun. The common mode impedance was clearly shown to be a function of the common mode current flow on the cables. Initially, in chapter 3, an EMC box was not used. The initial measurements as shown in fig.3.4 showed that its addition was beneficial. Although successful in restricting the common mode current flow, using an EMC box caused difficulty in that the antenna had to be positioned far enough from the EMC box to prevent reflections. In the process, the longer semi-rigid cables caused periodic behaviour in the results.

The use of a short pair of semi-rigid cables was shown to reduce results with periodicities and was sufficient and accurate where the differential impedance was concerned.

\section{Limitations of the method}

It was not investigated whether the reflections at the joining point between the semi-rigid cables and the SMA connectors caused errors of significant magnitude. The semi-rigid cables used for measurement had at least a $-20 \mathrm{~dB}$ or lower reflection coefficient at the joining point. This was assumed to be negligible. It is not known what the effect will be at much higher frequencies.

The method only provides information on the antenna impedance. It can not provide any information on other parameters such as the antenna radiation pattern, gain, axial ratio, etc. 


\section{References}

[1] V. Trifunovic and B. Jokanovic, "Review of printed marchand and double y baluns : Characteristics and application," IEEE Transactions on Microwave Theory and Techniques, vol. 42, pp. 1454-1462, Aug. 1994.

[2] R. Meys and F. Janssens, "Measuring the impedance of balanced antennas by an s-parameter method," IEEE Antennas and Propagation Magazine, vol. 40, Dec. 1998.

[3] G. C. Temes and J. W. LaPatra, Introduction to circuit synthesis and design. McGrawHill Inc., 1977. p. 327.

[4] D. A. Fricky, "Conversions between s,z,y,h, abcd, and t parameters which are valid for complex source and load impedances," IEEE Transactions on Microwave Theory and Techniques, vol. 42, pp. 208-209, Feb. 1994.

[5] IE3D Version 3.0. Zeland Software Inc, 39120 Argonaut Way, Suite 499, Fremont, CA, Jan. 1996.

[6] EMSS, Technopark, Stellenbosch 7600, South Africa, FEKO - User's Manual, Suite 2.3, January, 2000. www: http://www.feko.co.za.

[7] K. L. Su, Analog filters. Chapman \& Hall, 1996. London p.171.

[8] T. T. Wu and R. W. P. King, "The cylindrical antenna with nonreflecting resistive loading," IEEE Transactions Antennas and Propagation, vol. AP-13, pp. 369-373, May 1965. see Corrections [9].

[9] T. T. Wu and R. W. P. King, "The cylindrical antenna with nonreflecting resistive loading - corrections," IEEE Transactions Antennas and Propagation, vol. AP-13, p. p. 998, Nov. 1965. Original paper [8]. 


\section{Appendix}

\section{Derivation of the common mode and differential mode impedance as seen by a two port network analyzer.}

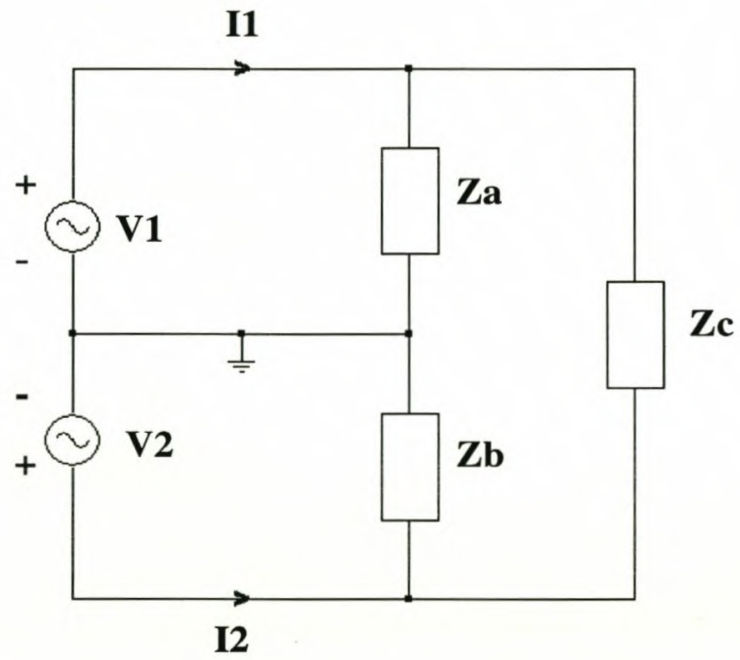

Figure 1: Circuit model for a complex antenna load when both ports of the network analyzer are connected.

From fig. 1 and using Y-parameters it is seen that

$$
\begin{aligned}
& I_{1}=Y 11 V_{1}+Y 12 V_{2} \\
& I_{2}=Y 21 V_{1}+Y 22 V_{2}
\end{aligned}
$$

To find Y11 source $V_{2}$ is short circuited :

$$
\begin{aligned}
Y 11 & =\left.\frac{I_{1}}{V_{1}}\right|_{V_{2}=0} \\
\Rightarrow Y 11 & =\frac{1}{Z_{a} \| Z_{c}}
\end{aligned}
$$

Similarly, to find Y22 source $V_{1}$ is short circuited :

$$
Y 22=\left.\frac{I_{2}}{V_{2}}\right|_{V_{1}=0}=\frac{1}{Z_{b} \| Z_{c}}
$$




$$
\begin{aligned}
Y 21 & =\left.\frac{I_{2}}{V_{1}}\right|_{V_{2}=0}=\frac{-1}{Z_{c}} \\
\Rightarrow Z c & =\frac{-1}{Y 21}
\end{aligned}
$$

Substitute (5) into (3) and solve for $Z_{a}$ :

$$
Z a=\frac{1}{Y 22+Y 21}
$$

Similarly, to find $Z_{b}$, substitute (5) into (4) and solve for $Z_{b}$ yielding

$$
Z b=\frac{1}{Y 11+Y 21}
$$

From fig. 1 it is seen that when the load or antenna is driven differentially, i.e. $\mathrm{Vc}=0$, the input impedance is $Z_{\text {diff }}=Z_{c} \|\left(Z_{a}+Z_{b}\right)$.

The common mode impedance of the antenna, i.e. $\mathrm{Vd}=0$, is obtained as follows:

With $\mathrm{Vd}=0$, the circuit in fig. 1 simplifies to the source Vc driving the impedances $\mathrm{Za}$ and $\mathrm{Zb}$ in parallel. Therefore

$Z_{\text {common }}=Z a \| Z b$. 